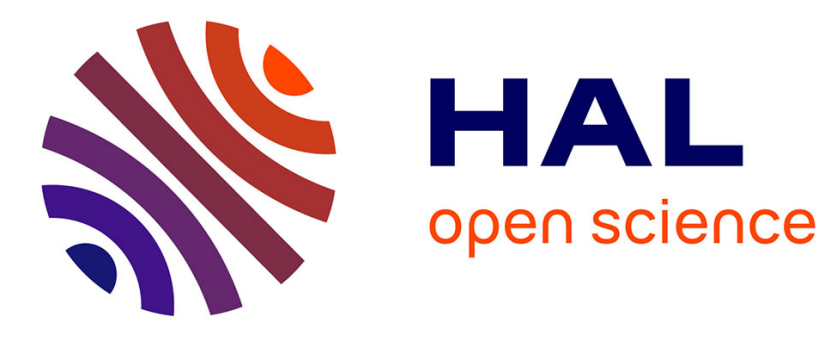

\title{
Model selection for hazard rate estimation in presence of censoring
}

Sandra Plancade

\section{To cite this version:}

Sandra Plancade. Model selection for hazard rate estimation in presence of censoring. Metrika, 2010, 74, pp.313-347. 10.1007/s00184-010-0305-9 . hal-02056881

\section{HAL Id: hal-02056881 \\ https://hal.science/hal-02056881}

Submitted on 4 Mar 2019

HAL is a multi-disciplinary open access archive for the deposit and dissemination of scientific research documents, whether they are published or not. The documents may come from teaching and research institutions in France or abroad, or from public or private research centers.
L'archive ouverte pluridisciplinaire HAL, est destinée au dépôt et à la diffusion de documents scientifiques de niveau recherche, publiés ou non, émanant des établissements d'enseignement et de recherche français ou étrangers, des laboratoires publics ou privés. 


\title{
Model selection for hazard rate estimation in presence of censoring
}

\author{
Sandra Plancade
}

Received: 13 May 2009 / Published online: 21 March 2010

(C) Springer-Verlag 2010

\begin{abstract}
This note presents an estimator of the hazard rate function based on right censored data. A collection of estimators is built from a regression-type contrast, in a general collection of linear models. Then, a penalised model selection procedure provides an estimator which satisfies an oracle inequality. In particular, we can prove that it is adaptive in the minimax sense on Hölder spaces.
\end{abstract}

Keywords Hazard C Censoring · Model selection · Adaptivity

\section{Introduction}

In medical follow-up and other subjects, the observation of a variable of interest, for example the lifetime of an individual, can be right censored. This means that we only observe the minimum of the lifetime and a variable called censoring time (for example the time when a patient leaves the medical program), which is supposed independent of the lifetime. We also observe if this minimum corresponds to the variable of interest or to the censoring time. More precisely, we consider a sample $\left(X_{i}\right)_{i=1, \ldots, n}$ of nonnegative variables, and a sample $\left(C_{i}\right)_{i=1, \ldots, n}$ of non-negative censoring times. Then we observe a sample $\left(T_{i}, \delta_{i}\right)_{i=1, \ldots n}$ with

$$
T_{i}=\min \left(X_{i}, C_{i}\right), \quad \delta_{i}=\mathbb{1}_{\left\{X_{i} \leq C_{i}\right\}}
$$

A function of interest in such a study is the hazard function of $X: \lambda(x)$, which represents the risk of death at a time $x$ knowing that the patient is alive until $x$. If we denote by $f_{X}(x)$ and $\bar{F}_{X}(x)=P\left[X_{1} \geq x\right]$ the density and the survival function of $X$, we

S. Plancade $(\varangle)$

MAP 5 UMR 8145, Université Paris Descartes, 45 rue des Saints Pères, 75006 Paris, France e-mail: sandra.plancade@mi.parisdescartes.fr 
have

$$
\lambda(x)=\frac{f_{X}(x)}{\bar{F}_{X}(x)} .
$$

A lot of papers are devoted to hazard rate estimation. In particular, it forms part of the most general study of counting processes (see Andersen et al. 1993). Two general methods can be drawn in the non parametric context that we only consider.

The first one consists in estimating $\lambda$ by a ratio of two estimators. The most obvious is $\widehat{f}_{X} / \widehat{F}_{X}$ where $\widehat{f}_{X}$ and $\widehat{F}_{X}$ are estimators of $f_{X}$ and $\bar{F}_{X}$. In general, $\bar{F}_{X}$ is replaced by the well known Kaplan Meier estimator of $\bar{F}_{X}$ (Kaplan and Meier 1958). Another decomposition of $\lambda$ is

$$
\lambda=\frac{f_{X} \bar{F}_{C}}{\bar{F}_{T}} .
$$

Indeed, $\bar{F}_{T}(x)=P[\{X \geq x\} \cap\{C \geq x\}]=\bar{F}_{X}(x) \bar{F}_{C}(x)$. The function $\psi(x)=$ $f_{X}(x) \bar{F}_{C}(x)$, called the subdensity of $X$, corresponds heuristically to the "density" of the observed variables $X_{i}$, in the sense that for every function $t: \mathbb{R}^{+} \rightarrow \mathbb{R}$ such that $t(0)=0$,

$$
\mathbb{E}\left[t\left(\delta_{i} T_{i}\right)\right]=\mathbb{E}\left[\delta_{i} t\left(X_{i}\right)\right]=\int t(x) \psi(x) d x
$$

As the $\left(\delta_{i}, X_{i}\right)$ are directly measured, $\psi$ is easier to estimate than $f_{X}$. Similarly, $\bar{F}_{T}$ is easier to estimate than $\bar{F}_{X}$. Indeed it can simply be replaced by the empirical survival function of the observed $\left(T_{i}\right)$. Patil (1993) proposes a kernel estimator of $\psi$ with a bandwidth selection and gets an estimator of $\lambda$ via Eq. 3. Antoniadis et al. (1999) use a wavelet decomposition but their estimator is not really adaptive as the optimal resolution of the wavelets depends on the regularity of $f_{X}$. Brunel and Comte (2005) build a projection estimator of $\psi$ by model selection in more general bases, and obtain an adaptive estimator.

Other estimators of $\lambda$ are based on the cumulative hazard $\Lambda(x)=-\log \left(\bar{F}_{X}(x)\right)$. One of the most frequently used estimator of $\Lambda$ is the Nelson-Aalen estimator (Nelson 1972). Obviously, we have

$$
\lambda(x)=\Lambda^{\prime}(x) .
$$

Yandell (1983) and Tanner and Wong (1983) build an estimator of $\lambda$ by differentiating the Nelson-Aalen estimator of $\Lambda$ with a delta-sequence method, and Muller and Wang (1994) introduce a variable bandwidth. Nielsen (2003) compares the numerical results from several variable bandwidth kernel estimators, and one of them is developed in Bagkavos and Patil (2009). Brunel and Comte (2008) propose a projection type estimator based on a approximation of cumulative hazard function. The method is very different from the one presented here, but leads also to an adaptive estimation procedure. 
Let us mention also the estimator of Reynaud-Bouret (2006) built by model selection in a set of random models, which is adaptive on Hölder spaces with regularity smaller than 1 .

The present note describes a regression type strategy, in a different spirit from other procedures. It leads to an adaptive estimator for the integrated squared risk on a set $[0, a]$ such that $P(T \geq a)$ is positive. The proofs are self contained (apart from the well-known Talagrand Inequality), and the key point is that the reference norm for the risk is chosen to be well suited to the problem.

The plan of the paper is the following. Section 2 presents the framework, and the main assumptions. The estimation procedure is described in Sect. 3, as well as the main result. But the estimator built in Sect. 3 brings into play unknown quantities, which are estimated in Sect. 4. The performance of these estimators on simulated data are presented in Sect. 5. The proofs are gathered in Sects. 6 and 7. Section 8 recalls classical deviation inequalities for empirical processes, and a technical algebra lemma.

\section{Presentation of the framework, assumptions and notations}

\subsection{Framework}

We consider a sample $\left(X_{1}, \ldots, X_{n}\right)$ of i.i.d. (independent identically distributed) non negative random variables with common survival function $\bar{F}_{X}(x)=P\left[X_{1} \geq x\right]$ and density $f_{X}$, and a sample $\left(C_{1}, \ldots, C_{n}\right)$ of i.i.d. non negative random variables with common survival function $\bar{F}_{C}$, independent of the $\left(X_{i}\right)$ 's. The variables of interest are the $\left(X_{i}\right)$ 's, but we only observe the sample $\left(\left(T_{1}, \delta_{1}\right), \ldots,\left(T_{n}, \delta_{n}\right)\right)$ defined in Eq. 1. The aim of this paper is to build an estimator of the hazard rate of $X_{1}$ given by Eq. 2, on a compact interval $A$ on which $\bar{F}_{T}=\bar{F}_{C} \bar{F}_{X}$ is lower bounded by a positive number, which is a classical assumption in such study. Theoretically, $A$ is a known compact interval independent of the data, even if practically it is chosen by looking at the data. More precisely, we consider the following assumptions.

A frame : We suppose that $\bar{F}_{T}$ is lower bounded on $A$ by $\bar{F}_{0}>0$, and that $\lambda$ is upper bounded by $\|\lambda\|_{\infty, A}=\sup _{x \in A} \lambda(x)<\infty$.

\subsection{Notations}

We define the following scalar products and norms on $L^{2}(A)$. For every $s, t \in L^{2}(A)$,

$$
\begin{aligned}
\langle s, t\rangle & =\int_{A} s(x) t(x) d x, \quad\|t\|^{2}=\int_{A} t^{2}(x) d x, \\
\langle s, t\rangle_{\bar{F}_{T}} & =\int_{A} s(x) t(x) \bar{F}_{T}(x) d x, \quad\|t\|_{\bar{F}_{T}}^{2}=\int_{A} t^{2}(x) \bar{F}_{T}(x) d x, \\
\langle s, t\rangle_{n} & =\frac{1}{n} \sum_{i=1}^{n} \int_{A} s(x) t(x) \mathbb{1}_{\left\{T_{i} \geq x\right\}} d x, \quad\|t\|_{n}^{2}=\frac{1}{n} \sum_{i=1}^{n} \int_{A} t^{2}(x) \mathbb{1}_{\left\{T_{i} \geq x\right\}} d x .
\end{aligned}
$$


Let $M$ be a matrix, we denote by $M^{t}$ the transpose of $M$. If $M$ is a square matrix, let $S p(M)$ be the set of the eigenvalues of $M$.

Let $\beta$ and $L$ be positive numbers, and $r$ the greatest integer smaller than $\beta$, we define the Hölder space $\mathcal{H}(\beta, L)$ on $A$,

$$
\mathcal{H}(\beta, L)=\left\{f: A \rightarrow \mathbb{R},\left|f^{(r)}(x)-f^{(r)}(y)\right| \leq L|x-y|^{\beta-r}, \forall x, y \in A\right\} .
$$

For every $x \in \mathbb{R}$, we denote by $E(x)$ the greatest integer smaller than or equal to $x$. For every subset $S$ of $\mathbb{R}$ we denote by $S^{c}$ the complementary of $S$ and by $\mathbb{1}_{S}$ the function which is equal to 1 on $S$ and to 0 on $S^{c}$.

All throughout the paper, $C_{i}$ denotes a universal numerical constant, and $C, C^{\prime}$ denote constants which depend on the given parameters of the problem and may change from one line to another.

\subsection{Collections of models}

We consider a collection $\mathcal{M}_{n}=\left\{S_{m}, m \in J_{n}\right\}$ of finite dimensional linear subsets of $L^{2}(A)$ with dimension $D_{m}=\operatorname{dim}\left(S_{m}\right) \leq n / \ln ^{2} n$. We suppose that $\mathcal{M}_{n}$ satisfies either Assumption $\mathbf{A}_{\mathbf{m o d}}^{(\mathbf{1})}$ or $\mathbf{A}_{\text {mod }}^{(\mathbf{2})}$.

$\mathbf{A}_{\text {mod }}^{(\mathbf{1})}$ : We suppose that $J_{n}=\left\{1, \ldots, N_{n}\right\}$ and

$$
S_{1} \subset S_{2} \subset \cdots \subset S_{N_{n}}
$$

Besides, there exists a constant $K$ such that for every model $S_{m}$, and for every $\left(\phi_{1}^{m}, \ldots, \phi_{D_{m}}^{m}\right)$ orthonormal basis of $S_{m}$ for the $L^{2}(A)$-norm,

$$
\sup _{x \in A} \sum_{k=1}^{D_{m}}\left(\phi_{k}^{m}(x)\right)^{2} \leq K^{2} D_{m} .
$$

$\mathbf{A}_{\text {mod }}^{(2)}$ : There exists a linear subset $S_{n}$ of $L^{2}(A)$ with dimension $N_{n} \leq n / \ln ^{2} n$ such that for every $m \in J_{n} S_{m} \subset S_{n}$, and the global space $S_{n}$ satisfies Eq. 5. Moroever, for every $c>0$, there exists a constant $C>0$ such that, for every $n \in \mathbb{N}^{*}$,

$$
\sum_{m \in J_{n}} \exp \left(-c \sqrt{D_{m}}\right) \leq C .
$$

Remark 1 1. Assumption $\mathbf{A}_{\text {mod }}^{(\mathbf{1})}$ is clearly stronger than Assumption $\mathbf{A}_{\text {mod }}^{(\mathbf{2})}$. Thus $\mathbf{A}_{\text {mod }}^{(2)}$ allows more irregular collections of models. For example, take $A=[0,1]$, let $I_{n}$ be the regular partition of $[0,1]$ of step $1 / N_{n}$, and $S_{n}$ the set of histograms on $[0,1]$ which are constant on $I_{n}$. Under Assumption $\mathbf{A}_{\text {mod }}^{(2)}$, the collection $\mathcal{M}_{n}$ can include any set of histograms $S_{m}$ based on a partition $I_{m}$ of $[0,1]$ composed of 
union of intervals from $I_{n}$, whereas Assumption $\mathbf{A}_{\mathbf{m o d}}^{(\mathbf{1})}$ only allows diadic regular set of histograms, namely

$$
S_{m}=\operatorname{Vect}\left\{\mathbb{1}_{\left[\frac{j-1}{D_{m}} \frac{j}{D_{m}}\right)}, j=1, \ldots D_{m}\right\}, \text { where } D_{m}=2^{m}, \quad m \in \mathbb{N}, \quad 2^{m} \leq N_{n}
$$

2. Condition Eq. 6 is equivalent to

$$
\sum_{D=1}^{N_{n}} \exp (-c \sqrt{D}) \operatorname{Card}\left(\left\{S_{m} \in \mathcal{M}_{n}, D_{m}=D\right\}\right) \leq C
$$

which restricts, for every $D \leq N_{n}$, the number of models $S_{m}$ in $\mathcal{M}_{n}$ of dimension $D_{m}=D$.

3. Under Assumption $\mathbf{A}_{\text {mod }}^{(\mathbf{1})}$, Eq. 6 is clearly satisfied since $\operatorname{Card}\left(\left\{S_{m} \in \mathcal{M}_{n}\right.\right.$, $\left.\left.D_{m}=D\right\}\right)=1$ for every $D \leq N_{n}$.

\section{Theoretical estimators}

The estimators built in this section bring into play unknown quantities, which are replaced by estimators in Sect. 4. In Sect. 3.1, we present a non adaptive procedure which provides an estimator $\widehat{\lambda}_{m}$ of $\lambda$ on each model $S_{m}$. The model selection procedure is described in Sect. 3.2, with two different penalties corresponding to the two Assumptions $\mathbf{A}_{\mathbf{m o d}}^{(\mathbf{1})}$ and $\mathbf{A}_{\mathbf{m o d}}^{(\mathbf{2})}$. Section 3.4 presents the main result.

\subsection{Non adaptive estimator}

We note that

$$
\lambda \mathbb{1}_{A}=\arg \min _{t \in L^{2}(A)}\|t-\lambda\|_{\bar{F}_{T}}^{2}=\arg \min _{t \in L^{2}(A)}\|t\|_{\bar{F}_{T}}^{2}-2\langle t, \lambda\rangle_{\bar{F}_{T}} .
$$

Now we build an estimator of $\|t\|_{\bar{F}_{T}}^{2}-2\langle t, \lambda\rangle_{\bar{F}_{T}}$. For every $t \in L^{2}(A), \mathbb{E}\left[\|t\|_{n}^{2}\right]=$ $\|t\|_{\bar{F}_{T}}^{2}$. In addition, for every $i=1, \ldots, n$,

$$
\begin{aligned}
\mathbb{E}\left[\delta_{i} t\left(T_{i}\right)\right] & =\mathbb{E}\left[\mathbb{E}\left[\delta_{i} t\left(T_{i}\right) \mid X_{i}\right]\right]=\mathbb{E}\left[t\left(X_{i}\right) \mathbb{E}\left[\mathbb{1}_{X_{i} \leq C_{i}} \mid X_{i}\right]\right] \\
& =\mathbb{E}\left[t\left(X_{i}\right) \bar{F}_{C}\left(X_{i}\right)\right]=\int_{A} t(x) \bar{F}_{C}(x) f_{X}(x) d x \\
& =\int_{A} t(x) \bar{F}_{C}(x) \bar{F}_{X}(x) \lambda(x) d x=\langle t, \lambda\rangle \bar{F}_{T} .
\end{aligned}
$$


Then, we set

$$
\gamma_{n}(t)=\|t\|_{n}^{2}-\frac{2}{n} \sum_{i=1}^{n} \delta_{i} t\left(T_{i}\right)
$$

for every $t \in L^{2}(A)$, and $\mathbb{E}\left[\gamma_{n}(t)\right]=\|t\|_{\bar{F}_{T}}-2\langle t, \lambda\rangle_{\bar{F}_{T}}$.

For every model $S_{m}$, let $\tilde{\lambda}_{m}=\arg \min _{t \in S_{m}} \gamma_{n}(t)$. Let $\left(\phi_{1}^{m}, \ldots, \phi_{D_{m}}^{m}\right)$ be an $L^{2}(A)$ orthonormal basis of $S_{m}$, then $\tilde{\lambda}_{m}=\sum_{k=1}^{D_{m}} \tilde{a}_{k}^{m} \phi_{k}^{m}$ where the $\left\{\tilde{a}_{k}^{m}\right\}$ 's satisfy

$$
\frac{\partial \gamma_{n}\left(\sum_{k=1}^{D_{m}} a_{k} \phi_{k}^{m}\right)}{\partial a_{k}^{m}}=0, \quad \forall k=1, \ldots D_{m} \quad \Leftrightarrow \quad \widehat{G}_{m} \tilde{A}_{m}=\widehat{V}_{m}
$$

with $\tilde{A}_{m}=\left(\tilde{a}_{1}, \ldots, \tilde{a}_{D_{m}}\right)^{t}$ and

$$
\widehat{G}_{m}=\left(\left\langle\phi_{k}^{m}, \phi_{k^{\prime}}^{m}\right\rangle_{n}\right)_{k, k^{\prime}=1, \ldots, D_{m}}, \quad \widehat{V}_{m}=\left(\frac{1}{n} \sum_{i=1}^{n} \delta_{i} \phi_{k}^{m}\left(T_{i}\right)\right)_{k=1, \ldots, D_{m}} .
$$

We note that $\tilde{\lambda}_{m}$ is uniquely defined iff $\widehat{G}_{m}$ is invertible. Thus we construct a set of high probability on which the spectrum of $\widehat{G}_{m}$ is lower bounded.

First of all, by Lemma 8.1,

$$
\min \left(\operatorname{Sp}\left(\widehat{G}_{m}\right)\right)=\min _{\left\{U \in \mathbb{R}^{D_{m}}, U^{t} U=1\right\}} U^{t} \widehat{G}_{m} U=\min _{\left\{t=\sum_{k=1}^{D_{m}} u_{k} \phi_{k}^{m},\|t\|=1\right\}}\|t\|_{n}^{2} .
$$

Besides, $\|\cdot\|_{n}$ is the empirical norm associated with $\|\cdot\|_{F_{T}}$, therefore the set

$$
\Delta_{1}=\left\{\left|\frac{\|t\|_{n}^{2}}{\|t\|_{F_{T}}^{2}}-1\right|<\frac{1}{4}, \forall t \in S_{n}\right\}
$$

has a probability close to 1 , which is proved in Proposition 6.3. On $\Delta_{1}, \min \left(\operatorname{Sp}\left(\widehat{G}_{m}\right)\right)=$ $\min _{\left\{t \in S_{m},\|t\|=1\right\}}(3 / 4)\|t\|_{\bar{F}_{T}}^{2} \geq(3 / 4) \bar{F}_{0}$, so the set

$$
\Delta_{2}^{t h}=\left\{\min \left(\operatorname{Sp}\left(\widehat{G}_{m}\right)\right) \geq \frac{3}{4} \bar{F}_{0}\right\}
$$

contains $\Delta_{1}$. Thus $P\left[\Delta_{2}^{t h}\right]$ is close to 1 , and $\widehat{G}_{m}$ is invertible on $\Delta_{2}^{t h}$. Finally, we define the estimator $\widehat{\lambda}_{m}=\sum_{k=1}^{D_{m}} \widehat{a}_{k} \phi_{k}^{m}$ with

$$
\widehat{A}_{m}=\left(\widehat{a}_{1}, \ldots, \widehat{a}_{D_{m}}\right)^{t}= \begin{cases}\widehat{G}_{m}^{-1} \widehat{V}_{m} & \text { on } \Delta_{2}^{t h} \\ 0 & \text { otherwise }\end{cases}
$$




\subsection{Adaptive estimators}

The non adaptive estimation procedure described in Sect. 3.1 provides a collection of estimators $\left\{\hat{\lambda}_{m}, m \in \mathcal{M}_{n}\right\}$, among which one is automatically selected by a penalised model selection procedure. We briefly present this strategy, developed by Birgé and Massart (1998). By Pythagore Theorem, for every model $S_{m}$ the risk of the estimator $\widehat{\lambda}_{m}$ is split in two terms.

$$
\mathbb{E}\left[\left\|\widehat{\lambda}_{m}-\lambda\right\|_{\bar{F}_{T}}^{2}\right]=\left\|\lambda-\lambda_{m}\right\|_{\bar{F}_{T}}^{2}+\mathbb{E}\left[\left\|\widehat{\lambda}_{m}-\lambda_{m}\right\|_{\bar{F}_{T}}^{2}\right]
$$

where $\lambda_{m}$ is the $\|\cdot\|_{\bar{F}_{T}}$-projection of $\lambda$ on $S_{m}$. The bias term $\left\|\lambda-\lambda_{m}\right\|_{\bar{F}_{T}}^{2}$ decreases when the model $S_{m}$ grows, whereas the term $\mathbb{E}\left[\left\|\widehat{\lambda}_{m}-\lambda_{m}\right\|_{\bar{F}_{T}}^{2}\right]$ has the order $D_{m} / n$ of a variance-type term, and increases with $D_{m}$. (Nevertheless, in our case, it is not exactly a variance term, for $\mathbb{E}\left[\widehat{\lambda}_{m}(x)\right] \neq \lambda_{m}(x)$.) Thus, the best model would be the one which generates the smallest risk, i.e. the one which realises the better trade-off between bias and variance.

The basic outline of model selection is to construct a data driven quantity which has the same order as the bias-variance sum (possibly up to a constant independent of $m$ ) and to select the model which minimises this quantity. On the one hand,

$$
\left\|\lambda-\lambda_{m}\right\|_{\bar{F}_{T}}^{2}=\left\|\lambda_{m}\right\|_{\bar{F}_{T}}^{2}-2\left\langle\lambda, \lambda_{m}\right\rangle_{\bar{F}_{T}}+\|\lambda\|_{\bar{F}_{T}}^{2} .
$$

The term $\left\|\lambda_{m}\right\|_{\bar{F}_{T}}^{2}-2\left\langle\lambda, \lambda_{m}\right\rangle_{\bar{F}_{T}}$ is estimated by $\gamma_{n}\left(\widehat{\lambda}_{m}\right)$ (see Eq. 7) and the term $\|\lambda\|_{\bar{F}_{T}}^{2}$ is independent of $m$. On the other hand, the variance term $\mathbb{E}\left[\left\|\widehat{\lambda}_{m}-\lambda_{m}\right\|_{\bar{F}_{T}}^{2}\right]$ is upper bounded by a deterministic term with order $D_{m} / n$, called the penalty. We do not explicitly prove this result here but a more general one (see Theorem 1 and Comment 1 hereafter).

We consider two penalties with order $D_{m} / n$, but with different constants.

$$
\operatorname{pen}_{1}^{t h}(m)=\frac{B K^{2}}{\bar{F}_{0}} \frac{D_{m}}{n}, \quad \operatorname{pen}_{2}^{t h}(m)=B\|\lambda\|_{\infty, A} \frac{D_{m}}{n}
$$

with $B>3$, and select the model

$$
\widehat{m}_{j}=\arg \min _{m \in J_{n}} \gamma_{n}\left(\widehat{\lambda}_{m}\right)+\operatorname{pen}_{j}^{t h}(m)
$$

for $j=1$ or 2 . We get two almost data-driven estimators of $\lambda: \widehat{\lambda}_{\widehat{m}_{1}}$ and $\widehat{\lambda}_{\widehat{m}_{2}}$. Each penalty corresponds to a set of assumptions. Penalty pen ${ }_{2}^{\text {th }}$ corresponds to Assumption $\mathbf{A}_{\text {mod }}^{(\mathbf{2})}$ so it works under both $\mathbf{A}_{\text {mod }}^{(\mathbf{1})}$ and $\mathbf{A}_{\text {mod }}^{(\mathbf{2})}$ (see Remark 1). Penalty pen ${ }_{2}^{\text {th }}$ only works under Assumption $\mathbf{A}_{\text {mod }}^{(\mathbf{1})}$, but is more computing-saving since $\bar{F}_{0}$ is estimated anyway to compute the non adaptive estimators (see $\Delta_{2}^{\text {th }}$ in Eq. 10). 
Remark 2 Actually, any constant $B>1$ could be allowed in the above penalties provided slight changes in the definition of $\Delta_{2}^{t h}$ are done, but we fix $B>3$ for simplicity's sake. This point is discussed more precisely in Sect. 6.5. Nevertheless, as $B$ tends to 1, the constants $C$ and $C^{\prime}$ involved in Theorem 1 tend to infinity.

\subsection{Result}

The following Theorem states the adaptivity of $\widehat{\lambda}_{\widehat{m}_{j}}$.

Theorem 1 Let $j=1$ or 2 . Under $\mathbf{A}_{\bmod }^{(\mathbf{j})}$ and $\mathbf{A}_{\text {frame, }}$

$$
\mathbb{E}\left[\left\|\widehat{\lambda}_{\widehat{m}_{j}}-\lambda\right\|_{\bar{F}_{T}}^{2}\right] \leq C \inf _{m \in J_{n}}\left\{\inf _{t \in S_{m}}\|t-\lambda\|_{\bar{F}_{T}}^{2}+\operatorname{pen}_{j}^{t h}(m)\right\}+\frac{C^{\prime}}{n}
$$

where $C$ is a numerical constant and $C^{\prime}$ depends on $\left(K, \bar{F}_{0},\|\lambda\|_{\infty}\right)$.

\section{Comments}

1. We do not study explicitly the risk of $\widehat{\lambda}_{m}$ for one model $S_{m}$ but a particular case of Eq. 12 when $\mathcal{M}_{n}$ is restricted to $\left\{S_{m}\right\}$ provides the following inequality.

$$
\mathbb{E}\left[\left\|\widehat{\lambda}_{m}-\lambda\right\|_{F_{T}}^{2}\right] \leq C^{\prime \prime}\left\{\inf _{t \in S_{m}}\|t-\lambda\|_{F_{T}}^{2}+\operatorname{pen}_{j}^{t h}(m)\right\}
$$

for $j=1$ or 2 .

2. Huber and MacGibbon (2004) prove that the minimax rate of convergence on the Hölder space $\mathcal{H}(\beta, L)$, for $\beta>0$ and $L>0$ is the classical rate $n^{-2 \beta /(2 \beta+1)}$. Besides, suppose that $\lambda \in \mathcal{H}(\beta, L)$,

$$
\inf _{t \in S_{m}}\|t-\lambda\|_{F_{T}} \leq \inf _{t \in S_{m}}\|t-\lambda\| \leq C(L, \beta) D_{m}^{-\beta}
$$

Thus for a model of dimension $D_{m^{*}}=E\left(n^{1 /(2 \beta+1)}\right)$,

$$
\mathbb{E}\left[\left\|\widehat{\lambda}_{m^{*}}-\lambda\right\|_{\bar{F}_{T}}^{2}\right] \leq C n^{-2 \beta /(2 \beta+1)} .
$$

So, for this choice $D_{m^{*}}, \widehat{\lambda}_{m^{*}}$ is optimal in the minimax sense on the space $\mathcal{H}(\beta, L)$. Thus, the collection $\mathcal{M}_{n}$ contains an estimator with optimal rate, but the choice of $D_{m^{*}}=n^{1 /(2 \beta+1)}$ is not accessible as $\beta$ is unknown.

3. The model selection procedure enables us to choose automatically such a model, without estimating $\beta$. More precisely, Inequality Eq. 12 (called an oracle inequality) shows that the risk bound of $\widehat{\lambda}_{\widehat{m}_{j}}$ has same order as the risk of the best estimator among the collection $\left\{\widehat{\lambda}_{m}, m \in \mathcal{M}_{n}\right\}$. In particular, $\widehat{\lambda}_{\widehat{m}_{j}}$ reaches the minimax rate of convergence $n^{2 \beta /(2 \beta+1)}$ over all Hölder classes $\mathcal{H}(\beta, L)$ for $\beta>0, L>0$. 


\section{Data-driven estimators}

The estimators presented in this section are similar to the ones of Sect. 3, but the unknown quantities $\bar{F}_{0}$ and $\|\lambda\|_{\infty, A}$ are replaced by estimators.

\subsection{Estimator of $\bar{F}_{0}$}

$\bar{F}_{0}$ is the lower bound of $\bar{F}_{T}$ on $A=[0, a]$, so $\bar{F}_{0}=\bar{F}_{T}(a)$. Thus a natural estimator of $\bar{F}_{0}$ would be the value of the empirical survival function in a. In order to force the estimator of $\bar{F}_{0}$ to be lower bounded, we define:

$$
\widehat{F}_{0}=\max \left(\alpha_{n}, \frac{1}{n} \sum_{i=1}^{n} \mathbb{1}_{\left\{T_{i} \geq a\right\}}\right), \quad \text { where } \alpha_{n}=1 / \sqrt{n}
$$

Let

$$
\Delta_{2}=\left\{\min \left(\operatorname{Sp}\left(\widehat{G}_{m}\right)\right) \geq \frac{3}{5} \widehat{F}_{0}\right\} \quad \text { and } \quad \Delta_{3}=\left\{\frac{3}{4} \bar{F}_{0} \leq \widehat{F}_{0} \leq \frac{5}{4} \bar{F}_{0}\right\}
$$

The following result holds.

\section{Proposition 4.1}

$$
\Delta_{1} \cap \Delta_{3} \subset \Delta_{2}^{t h} \cap \Delta_{3} \subset \Delta_{2} \cap \Delta_{3}
$$

Proof of Proposition 4.1 According to Sect. 3.1,

$$
\Delta_{1} \subset \Delta_{2}^{t h} \Rightarrow \Delta_{1} \cap \Delta_{3} \subset \Delta_{2}^{t h} \cap \Delta_{3} .
$$

Moreover, on the set $\Delta_{2}^{t h} \cap \Delta_{3}$, according to Lemma 8.1,

$$
\min \left(\operatorname{Sp}\left(\widehat{G}_{m}\right)\right)=\inf _{\left\{t \in S_{m}, t \neq 0\right\}} \frac{\|t\|_{n}^{2}}{\|t\|^{2}} \geq \frac{3}{4} \bar{F}_{0} \geq \frac{3}{4} \times \frac{4}{5} \widehat{F}_{0}=\frac{3}{5} \widehat{F}_{0}
$$

hence $\Delta_{2}^{t h} \cap \Delta_{3} \subset \Delta_{2} \cap \Delta_{3}$.

\subsection{Estimator of $\|\lambda\|_{\infty, A}$}

Let $v=\|\lambda\|_{\infty, A}$. Let $D=E\left(n^{\gamma}\right)$ be a middle-sized model with $0<\gamma<1$, and $S_{D}=\operatorname{Vect}\left(\varphi_{1}^{D}, \ldots, \varphi_{D}^{D}\right)$ the set of piecewise constant functions on $[0, a]$ with $\varphi_{j}^{D}=\sqrt{D / a} \mathbb{1}_{[a(j-1) / D, a j / D)}$. Let $\widehat{\lambda}_{D}=\arg \min _{t \in S_{D}} \gamma_{n}(t)$. As the basis functions $\left(\varphi_{j}^{D}\right)$ have disjoint supports, the matrix $\widehat{G}_{D}$ of the scalar product $\langle.,\rangle_{n}$ in the basis 
$\left(\varphi_{1}^{D}, \ldots, \varphi_{D}^{D}\right)$ is diagonal, with diagonal coefficients $\left(\left\|\varphi_{j}^{D}\right\|_{n}^{2}\right)_{j=1, \ldots, D}$. On $\Delta_{2}$,

$$
\left\|\varphi_{j}^{D}\right\|_{n}^{2} \geq \frac{3}{5} \widehat{F}_{0} \geq \frac{3}{5} \alpha_{n}>0
$$

thus $\widehat{G}_{D}$ is invertible. Let $\widehat{\lambda}_{D}=\sum_{j=1, \ldots, D} \widehat{a}_{j} \varphi_{j}^{D}$ where

$$
\widehat{a}_{j}= \begin{cases}\frac{(1 / n) \sum_{i=1}^{n} \delta_{i} \varphi_{j}^{D}\left(T_{i}\right)}{\left\|\varphi_{j}^{D}\right\|_{n}^{2}} & \text { on } \Delta_{2} \\ 0 & \text { otherwise. }\end{cases}
$$

Let $\widehat{v}_{n}=\|\widehat{\lambda}\|_{\infty}$, then

$$
\widehat{v}_{n}=\sqrt{\frac{D}{a}} \max _{j=1 \ldots, D} \widehat{a}_{j} .
$$

Besides, let $\lambda_{D}$ be the $\|\cdot\|_{\bar{F}_{T}}$-projection of $\lambda$ on $S_{D}$,

$$
\lambda_{D}=\sum_{j=1, \ldots, D} a_{j} \varphi_{j}^{D} \quad \text { where } \quad a_{j}=\frac{\int_{A} \varphi_{j}^{D}(x) \lambda(x) \bar{F}_{T}(x) d x}{\left\|\varphi_{j}^{D}\right\|_{\bar{F}_{T}(x)}^{2}} .
$$

Finally, we define the following set whose probability is close to 1 (see Proposition 7.3),

$$
\Delta_{4}=\left\{\frac{3}{4} v \leq \widehat{v}_{n} \leq \frac{5}{4} v\right\}
$$

\subsection{Data-driven estimator}

Let $S_{m}$ be a model of the collection $\mathcal{M}_{n}$. We follow a procedure similar to the one described in Sect. 3.1, but now the set $\Delta_{2}^{t h}$ is replaced by $\Delta_{2}$. Let $\widehat{\lambda}_{m}=\sum_{k=1}^{D_{m}} \widehat{a}_{k}^{m} \phi_{k}$ where $\widehat{A}_{m}=\left(\widehat{a}_{1}^{m}, \ldots, \widehat{a}_{D_{m}}^{m}\right)^{t}=\widehat{G}_{m}^{-1} \widehat{V}_{m}$ on $\Delta_{2}$, and 0 otherwise, and $\widehat{G}_{m}$ and $\widehat{V}_{m}$ are defined in (8). Moreover, let

$$
\operatorname{pen}_{1}(m)=\frac{B K^{2}}{\widehat{F}_{0}} \frac{D_{m}}{n}, \quad \operatorname{pen}_{2}(m)=B \widehat{v}_{n} \frac{D_{m}}{n}
$$

with $B>15 / 4$. Finally we consider the estimators $\widehat{\lambda}_{\widehat{m}_{1}}$ and $\widehat{\lambda}_{\widehat{m}_{2}}$ where

$$
\widehat{m}_{j}=\arg \min _{m \in J_{n}} \gamma_{n}\left(\widehat{\lambda}_{m}\right)+\operatorname{pen}_{j}(m)
$$

for $j=1$ or 2 . 


\subsection{Results}

Now our estimators are completely data-driven when $B$ is chosen, and we can generalize Theorem 1 as follows.

Theorem 2 Let $j=1$ or 2 . Assume that $\mathbf{A}_{\text {mod }}^{(\mathbf{j})}$ and $\mathbf{A}_{\text {frame hold, as well as the fol- }}$ lowing condition:

$$
\left\|\lambda-\lambda_{D}\right\|_{\infty} \leq \frac{v}{8}
$$

Then

$$
\mathbb{E}\left[\left\|\widehat{\lambda}_{\widehat{m}_{j}}-\lambda\right\|_{\bar{F}_{T}}^{2}\right] \leq C \inf _{m \in J_{n}}\left[\inf _{t \in S_{m}}\|t-\lambda\|_{\bar{F}_{T}}^{2}+\operatorname{pen}_{j}^{t h}(m)\right]+\frac{C^{\prime}}{n}
$$

where $C$ is a numerical constant and $C^{\prime}$ depends on $\left(K, \bar{F}_{0},\|\lambda\|_{\infty}, a\right)$.

Remark 3 (1) If $\lambda$ is in the Hölder space $\mathcal{H}(\beta, L)$ for some $\beta \in] 0,1[, L>0$, then Eq. 14 is satisfied for $n$ large enough. In fact, let $y \in A$ :

$$
\begin{aligned}
\left|\lambda(y)-\lambda_{D}(y)\right| & =\left|\lambda(y)-\frac{(D / a) \int_{a(j-1) / D}^{a j / D} \lambda(x) \bar{F}_{T}(x) d x}{(D / a) \int_{a(j-1) / D}^{a j / D} \bar{F}_{T}(x) d x}\right| \\
& =\frac{\left|(D / a) \int_{a(j-1) / D}^{a j / D} \lambda(y) \bar{F}_{T}(x) d x-(D / a) \int_{a(j-1) / D}^{a j / D} \lambda(x) \bar{F}_{T}(x) d x\right|}{(D / a) \int_{a(j-1) / D}^{a j / D} \bar{F}_{T}(x) d x} \\
& \leq \frac{\int_{a(j-1) / D}^{a j / D}|\lambda(y)-\lambda(x)| \bar{F}_{T}(x) d x}{\int_{a(j-1) / D}^{a j / D} \bar{F}_{T}(x) d x} \leq \frac{L}{D^{\beta}} .
\end{aligned}
$$

Comments of Sect. 3.3 hold, thus the adaptive estimators are minimax over Hölder spaces.

(2) As notified in Remark 2, $B$ could be choosen as any numerical constant, provided that it is greater than 1 .

\section{Numerical examples}

In this section, we present the performance of the model selection estimator on simulated data. For sake of simplicity, we suppose that the parameters $\bar{F}_{0}$ and $\|h\|_{\infty, A}$ are known, which corresponds to the estimators described in Sect. 2. Besides, preliminary numerical studies prove that replacing these parameters by estimators hardly affects the result. We consider two collections of models of functions supported on $[0, a]$ for some $a>0$. 
1. For $m \in \mathbb{N}^{*}$, let

$$
S_{m}^{1, a}=\operatorname{vect}\left(\left\{\phi_{1, m}^{(1)}: x \rightarrow 1\right\} \cup\left\{\phi_{k, m}^{(1)}: x \rightarrow \sqrt{2 / a} \cos (\pi(k-1) x / a), k=2, \ldots, m\right\}\right) .
$$

This basis is not exactly the classical trigonometric basis: only cosine elements are used, and instead of $\cos (2 \pi k x / a)$ and $\sin (2 \pi k x / a)$ we consider $\cos (\pi k x / a)$. This model is based on the following observation. Let $t$ be a function defined on $[0, a]$, $t$ can be extended as an even function $t^{*}$ on $[-a, a]$ by setting $t^{*}(-x)=t(x)$. Thus by classical Fourier analysis, $t^{*}$ can be expanded in the basis $\{\cos (\pi k x / a)\}$ since the sine Fourier coefficients of $t^{*}$ vanishes, and so does $t$. (See Efromovich 2007, Sect. 3, Remark 1 for more details.)

2. For $m \in \mathbb{N}^{*}$, let

$$
S_{m}^{2, a}=\operatorname{vect}\left(\left\{\phi_{k, m}^{(2)}: x \rightarrow \sqrt{m / a} \mathbb{1}_{[a(k-1) / m, a k / m)}(x), k=1, \ldots, m\right\}\right)
$$

be the set of histograms of step $a / m$.

Each simulation run is constructed as follows.

- Sequences $\left(X_{1}, \ldots, X_{n}\right)$ and $\left(C_{1}, \ldots, C_{n}\right)$ are simulated following one of these distributions.

(i) $X_{i}$ has a bimodal density defined by $f_{X}=0.8 u+0.2 v$ where $u$ is the density of $\exp (W / 2)$ with $W \sim \mathcal{N}(0,1)$ and $v$ is the density of a gaussian distribution with mean 2 and variance 0.17 . The $\left(C_{i}\right)$ 's are generated from an exponential distribution with mean 2.5.

(ii) $X_{i}$ is generated from a gamma distribution with shape parameter 5 and scale 1 . The $\left(C_{i}\right)$ 's are generated from an exponential distribution with mean 6 .

The mean of the $\left(C_{i}\right)$ 's distribution is numerically choosen such that $\mathbb{E}\left[\frac{1}{n}\left(\sum_{i=1}^{n} \mathbb{1}_{\left\{X_{i} \geq C_{i}\right\}}\right)\right]=0.4$.

- We compute the sample $\left(T_{i}, \delta_{i}\right)_{i=1, \ldots, n}$ where $T_{i}=\min \left(X_{i}, C_{i}\right)$ and $\delta_{i}=\mathbb{1}_{\left\{X_{i} \leq C_{i}\right\}}$.

- We consider either the collection $\left\{S_{m}^{1, a}, m=1, \ldots, \sqrt{n}\right\}$ or $\left\{S_{m}^{2, a}, m=\right.$ $1, \ldots, \sqrt{n}\}$ supported on $[0, a]$ with: (i) $a=3$ (ii) $a=10$. In numerical computing we can restrict ourselves to models $m$ smaller than $\sqrt{n}$ without altering the result, since in practice the selected model is much smaller than $\sqrt{n}$. For every $m \in\{1, \ldots, \sqrt{n}\}$, we compute the matrix

$$
\widehat{G}_{m}=\left(\frac{1}{n} \sum_{i=1}^{n} \int_{0}^{a} \phi_{k, m}^{(j)}(x) \phi_{k^{\prime}, m}^{(j)}(x) \mathbb{1}_{\left\{x \leq T_{i}\right\}} d x\right)_{k, k^{\prime}=1, \ldots, m},
$$

the column vector

$$
\widehat{V}_{m}=\left[\frac{1}{n} \sum_{i=1}^{n} \delta_{i} \phi_{k, m}^{(j)}\left(T_{i}\right)\right]_{k=1, \ldots, m}
$$


and the coefficient vector

$$
\widehat{A}_{m}= \begin{cases}\widehat{G}_{m}^{-1} \widehat{V}_{m} & \text { if } \max \left(\operatorname{Sp}\left(\widehat{G}_{m}\right)\right) \geq \bar{F}_{0} / 4 \\ 0 & \text { otherwise }\end{cases}
$$

where $\bar{F}_{0}=\bar{F}_{T}(a)$ and $\bar{F}_{T}$ is the survival function of $T$.

- We select the model $\widehat{m} \in\{1, \ldots \sqrt{n}\}$ which minimizes

$$
\widehat{A}_{m}^{t} \widehat{G}_{m} \widehat{A}_{m}-2 \widehat{A}_{m}^{t} \widehat{V}_{m}+\frac{3}{2}\|h\|_{\infty, A} \frac{m}{n}=-\widehat{A}_{m}^{t} \widehat{V}_{m}+\frac{3}{2}\|h\|_{\infty, A} \frac{m}{n}
$$

where $\|h\|_{\infty, A}$ is the maximum of $h$ on $A=[0, a]$.

- Let $I$ be the set of $100 a+1$ equispaced points in $[0, a]$. For every $x \in I$, we compute

$$
\widehat{h}_{\widehat{m}}(x)=\sum_{k=0}^{\widehat{m}-1} \widehat{a}_{k, \widehat{m}} \phi_{k, \widehat{m}}^{(j)}(x)
$$

where $\widehat{A}_{m}=\left(\widehat{a}_{0, m}, \ldots, \widehat{a}_{m-1, m}\right)^{t}$. We plot the set of points $\left\{\left(x, \widehat{h}_{\widehat{m}}(x)\right), x \in I\right\}$.

\subsection{Bimodal distribution}

Consider the model (i). Figure 1 illustrates the performance of the model selection estimator in trigonometric basis for sample sizes $n=200,500$ and 1,000, and Fig. 2 presents a beam of 20 estimators for $n=500$. We notice that the estimation is bad at the end of the interval, which is classically observed in hazard rate estimation. Besides, this behaviour is consistent with the theoretical aspect. Indeed $\bar{F}_{T}$ is decreasing, so the $\|\cdot\|_{\bar{F}_{T}}$-risk considered in Sects. 2 and 3 puts more weight on the beginning of the interval and the bad estimation at the end of the interval has less influence on the risk $\left\|\widehat{h}_{\widehat{m}}-h\right\|_{\bar{F}_{T}}$. Thus, similarly to Brunel and Comte (2005) and Antoniadis et al. (1999), we also present in Fig. 2 the beam of curves of 20 estimators restricted to the interval $[0,2]$. We note that estimation is much better than on the full interval $[0,3]$.

\subsection{Gamma distribution}

Consider the model (ii). Figure 3 illustrates the performance of the model selection estimator in histogram basis for sample sizes $n=200,500$ and 1,000. Similarly to the bimodal model, we present in Fig. 4 a beam of 20 estimators for $n=1,000$ on $[0,10]$ and the same beam restricted to the interval $[0,6]$. 


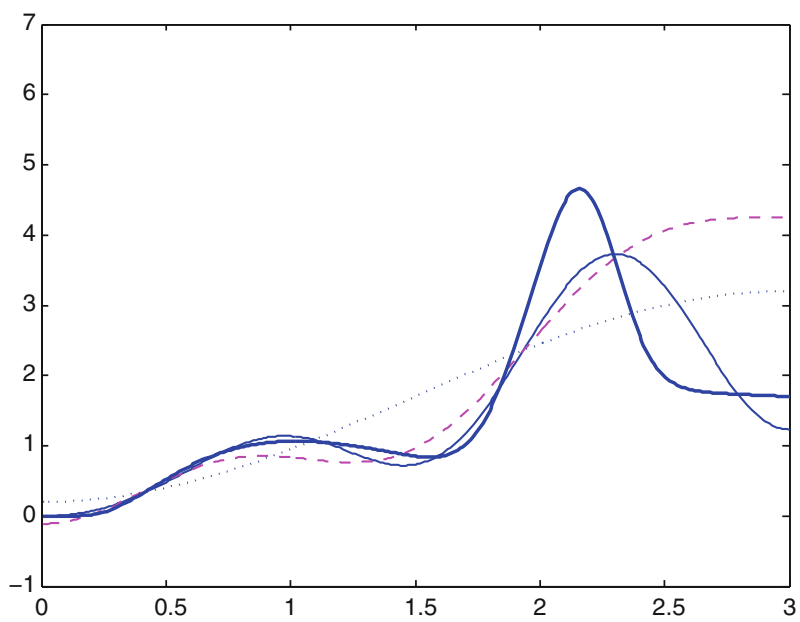

Fig. 1 Model selection estimator in trigonometric basis and true $h$ (thick line) for $n=200$ (small dotted line), $n=500$ (large dotted line) and $n=1,000$ (solid line) from the bimodal distribution
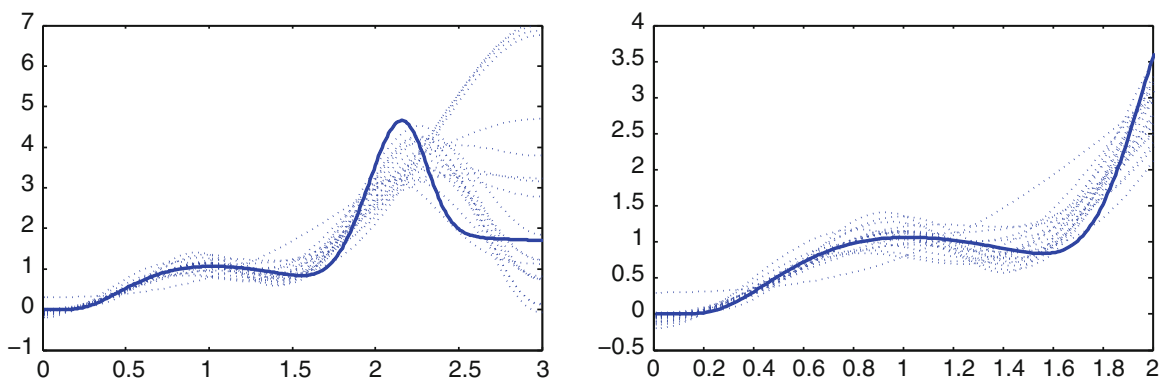

Fig. 2 Beam of 20 estimators in trigonometric basis for $n=500$ (small dotted line) from the bimodal distribution and true $h$ (thick line)

\subsection{Numerical results}

Examples (i) and (ii) have been studied in Antoniadis et al. (1999), Reynaud-Bouret (2006) and Brunel and Comte (2005). Antoniadis et al. (1999) use wavelet methods, Reynaud-Bouret (2006) builds an histogram estimator, and Brunel and Comte (2005) also use model selection but with a different contrast. The authors study the squared integrated risk of the estimator $\widehat{h}$ : they draw a large number $L$ of replications $\left\{\left(T_{i}^{l}, \delta_{i}^{l}\right)_{i=1, \ldots, n}, l=1, \ldots, L\right\}$, and compute an estimator $\widehat{h}^{l}$ for each replication. Then the MSE is the quantity

$$
\frac{1}{L} \sum_{l=1}^{L}\left[\frac{1}{J} \sum_{j=1}^{J}\left(\widehat{h}^{l}\left(t_{j}\right)-h\left(t_{j}\right)\right)^{2}\right]
$$




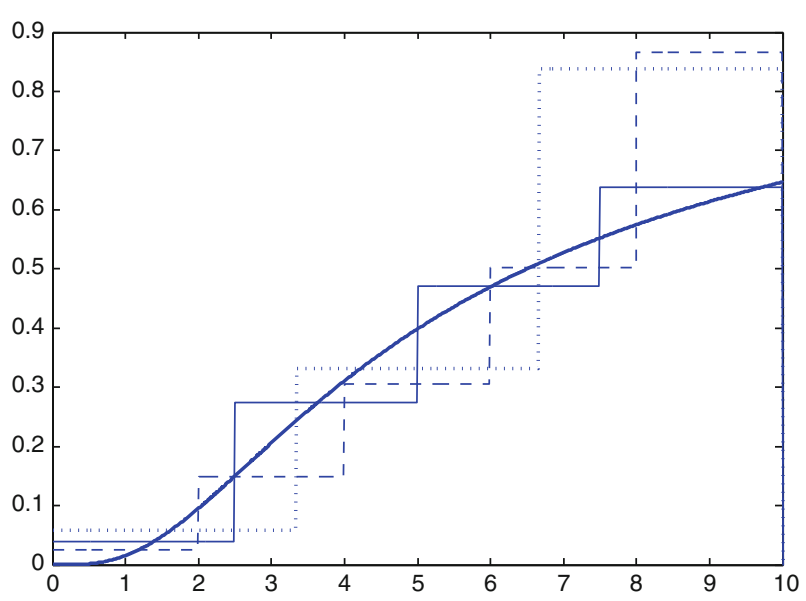

Fig. 3 Model selection estimator in trigonometric basis and true $h$ (thick line) for $n=200$ (small dotted line), $n=500$ (large dotted line) and $n=1,000$ (solid line) from the bimodal distribution
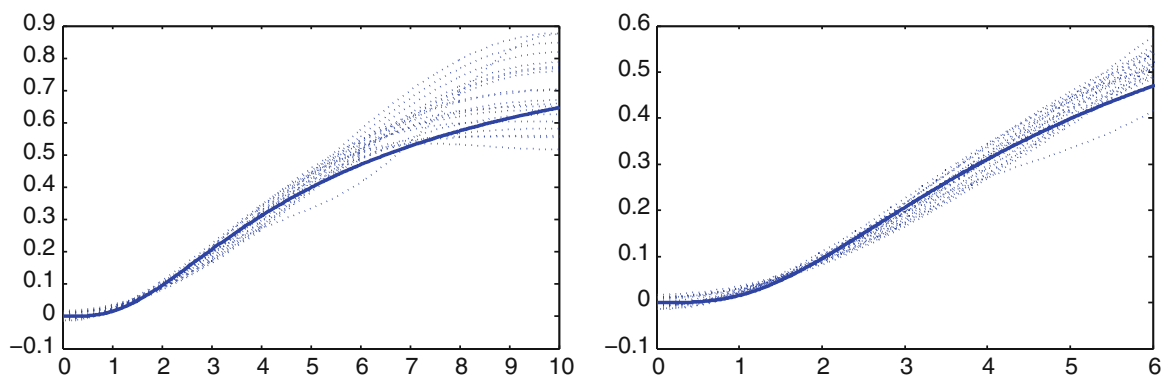

Fig. 4 Beam of 20 estimators in histogram basis for $n=500$ (small dotted line) from the gamma distribution and true $h$ (thick line)

where the $\left(t_{j}\right)$ 's are regularly spaced points in the interval $\left[0, \max \left(T_{i}\right)\right]$. It turns out to be difficult to compare their estimators with our since we do not estimate $h$ on the same interval. Nevertheless, they also compute an error MSE2 similarly to MSE but on a restricted interval $[0, b]$ with $b=6$ in the gamma case and $b=2$ in the bimodal case. More precisely, MSE2 is equal to Eq. 15 where the $\left(t_{k}\right)$ 's are regularly spaced points in the interval $[0, b]$. In our examples, we take $100 \mathrm{a}+1$ equispaced points in $[0$, a] and $L=500$ replications.

Performances of the three above-mentionned estimators for the MSE2 are gathered in Table 1, whereas Table 2 shows the performance of our estimator in bimodal and gamma case.

We notice that our estimator provides slightly better results for the MSE2 in the gamma model, and slightly less good results in the bimodal model. 
Table 1 Results of MSE2 for the estimators of Antoniadis et al. (1999), Brunel and Comte (2005) and Reynaud-Bouret (2006), for bimodal and gamma models

\begin{tabular}{|c|c|c|c|c|c|c|}
\hline \multirow[t]{2}{*}{ Model } & \multicolumn{2}{|c|}{ Antoniadis et al. } & \multicolumn{2}{|c|}{ Brunel and Comte } & \multicolumn{2}{|c|}{ Reynaud-Bouret } \\
\hline & Gamma & Bimodal & Gamma & Bimodal & Gamma & Bimodal \\
\hline$n=200$ & 0.0025 & 0.048 & 0.0023 & 0.1068 & 0.0032 & 0.150 \\
\hline$n=500$ & 0.0016 & 0.032 & 0.0013 & 0.0408 & 0.0012 & 0.051 \\
\hline
\end{tabular}

Table 2 Results of MSE2 for our estimator, for bimodal and gamma models

\begin{tabular}{llc}
\hline Model & Gamma & Bimodal \\
\hline$n=200$ & 0.0019 & 0.172 \\
$n=500$ & 0.0009 & 0.080 \\
\hline
\end{tabular}

Table 3 Mean and empirical variance of $\widehat{m}$ for bimodal and gamma models

\begin{tabular}{llllll}
\hline & \multicolumn{2}{l}{ Gamma } & & & \multicolumn{2}{l}{ Bimodal } \\
\cline { 2 - 3 } & $\overline{\overline{\widehat{m}}}$ & $\operatorname{Var}(\widehat{m})$ & & $\overline{\widehat{m}}$ & $\operatorname{Var}(\widehat{m})$ \\
\hline$n=200$ & 2.26 & 0.51 & 3.33 & 2.50 \\
$n=500$ & 2.50 & 0.65 & 5.00 & 2.85 \\
$n=1,000$ & 2.89 & 0.65 & 5.49 & 2.89 \\
\hline
\end{tabular}

We also compute the mean and the empirical variance of the selected model for $L=500$ replications, namely,

$$
\overline{\widehat{m}}=\frac{1}{L} \sum_{l=1}^{L} \widehat{m}^{l} \quad \text { and } \quad \operatorname{Var}(\widehat{m})=\frac{1}{L} \sum_{l=1}^{L}\left(\widehat{m}^{l}-\overline{\widehat{m}}\right)^{2}
$$

where $\widehat{m}^{l}$ is the selected model from the $l$ th sample. The results gathered in Table 3 indicate that the model selection algorithm really selects various values of $\widehat{m}$ for the different runs (see the variance of the chosen $\widehat{m}$ 's), and thus adapts really to the data.

Besides, our estimator is quite fast-computing. (For example, the running time for the MSE2 computed with $L=500$ replications of a sample of size $n=500$ is a few seconds on a personnal computer.)

\section{Proof of Theorem 1}

The following Propositions are intermediate results to prove Theorem 1. Assume that A frame holds. 
Proposition 6.1 Let $j=1$ or 2. Under $\mathbf{A}_{\text {mod }}^{(\mathbf{j})}$,

$$
\mathbb{E}\left[\left\|\widehat{\lambda}_{\widehat{m}_{j}}-\lambda\right\|_{F_{T}}^{2} \mathbb{1}_{\Delta_{1}}\right] \leq C \inf _{m \in J_{n}}\left[\inf _{t \in S_{m}}\|t-\lambda\|_{\bar{F}_{T}}^{2}+\operatorname{pen}_{j}^{t h}(m)\right]+\frac{C^{\prime}}{n}
$$

where $C$ is a numerical constant and $C^{\prime}$ depends on $\left(K, \bar{F}_{0},\|\lambda\|_{\infty}\right)$.

Proposition 6.2 For every model $S_{m} \in \mathcal{M}_{n}$,

$$
\left\|\widehat{\lambda}_{m}-\lambda\right\|_{\bar{F}_{T}} \leq \frac{2 K \sqrt{N_{n}}}{\bar{F}_{0}}+\|\lambda\|_{\bar{F}_{T}} \text { a.s. }
$$

Proposition 6.3 Assume that $\mathbf{A}_{\bmod }^{(\mathbf{1})}$ or $\mathbf{A}_{\text {mod }}^{(2)}$ holds, then

$$
P\left[\Delta_{1}^{c}\right] \leq 2 \exp \left(-C_{1} \bar{F}_{0}^{2} \frac{n}{N_{n}}\right)
$$

where $C_{1}$ is a numerical constant.

\subsection{Proof of Theorem 1}

Under $\mathbf{A}_{\text {mod }}^{(\mathbf{j})}, N_{n} \leq n /(\ln n)^{2}$ so according to Proposition 6.2 and 6.3,

$$
\begin{aligned}
\mathbb{E}\left[\left\|\widehat{\lambda}_{\widehat{m}_{j}}-\lambda\right\|_{F_{T}}^{2} \mathbb{1}_{\Delta_{1}^{c}}\right] \leq & \left(\frac{2 K \sqrt{N_{n}}}{\bar{F}_{0}}+\|\lambda\|_{F_{T}}\right) \\
& \times \exp \left[-C_{1} \bar{F}_{0} \frac{n}{N_{n}}\right] \leq C n \exp \left[-C_{1} \bar{F}_{0}(\ln n)^{2}\right] \\
= & C n\left[\exp \left(-C_{1} \bar{F}_{0} \ln n\right)\right]^{\ln n} \\
= & C n\left[n^{-C_{1} \bar{F}_{0}}\right]^{\ln n}=C n^{1-C_{2} \bar{F}_{0} \ln n} \leq \frac{C^{\prime}}{n}
\end{aligned}
$$

which, together with Proposition 6.1, ends the proof of Theorem 1.

\subsection{Proof of Proposition 6.1}

Let $j=1$ or 2 . To simplify notations, we denote $\operatorname{pen}(m)=\operatorname{pen}_{j}^{t h}(m)$ and $\widehat{m}=\widehat{m}_{j}$. Let $S_{m}$ be a model in the collection $\mathcal{M}_{n}$ and $\lambda_{m}$ be any function in $S_{m}$. On the set $\Delta_{2}^{t h}$,

$$
\gamma_{n}\left(\widehat{\lambda}_{\widehat{m}}\right)+\operatorname{pen}(\widehat{m}) \leq \gamma_{n}\left(\lambda_{m}\right)+\operatorname{pen}(m) .
$$

Thus,

$$
\left\|\widehat{\lambda}_{\widehat{m}}\right\|_{n}^{2}-\left\|\lambda_{m}\right\|_{n}^{2} \leq \operatorname{pen}(m)-\operatorname{pen}(\widehat{m})+\frac{2}{n} \sum_{i=1}^{n} \delta_{i}\left(\widehat{\lambda}_{\widehat{m}}-\lambda_{m}\right)\left(T_{i}\right) .
$$


Besides, $\left.\left\|\widehat{\lambda}_{\widehat{m}}-\lambda_{m}\right\|_{n}^{2}=\left\|\widehat{\lambda}_{\widehat{m}}\right\|_{n}^{2}+\left\|\lambda_{m}\right\|_{n}^{2}-2 \widehat{\lambda}_{\widehat{m}}, \lambda_{m}\right\rangle_{n}$, so

$$
\begin{aligned}
\left\|\widehat{\lambda}_{\widehat{m}}-\lambda_{m}\right\|_{n}^{2} & \left.\leq \operatorname{pen}(m)-\operatorname{pen}(\widehat{m})+\frac{2}{n} \sum_{i=1}^{n} \delta_{i}\left(\widehat{\lambda}_{\widehat{m}}-\lambda_{m}\right)\left(T_{i}\right)-2 \widehat{\left\langle\lambda_{\widehat{m}}\right.}, \lambda_{m}\right\rangle_{n}+2\left\|\lambda_{m}\right\|_{n}^{2} \\
& \left.=\operatorname{pen}(m)-\operatorname{pen}(\widehat{m})-2 \widehat{\left\langle\lambda_{\widehat{m}}\right.}-\lambda_{m}, \lambda_{m}\right\rangle_{n}+\frac{2}{n} \sum_{i=1}^{n} \delta_{i}\left(\widehat{\lambda}_{\widehat{m}}-\lambda_{m}\right)\left(T_{i}\right) \\
& \left.=\operatorname{pen}(m)-\operatorname{pen}(\widehat{m})-2 \widehat{\left\langle\lambda_{\widehat{m}}\right.}-\lambda_{m}, \lambda_{m}-\lambda\right\rangle_{n}+2 v_{n}\left(\widehat{\lambda}_{\widehat{m}}-\lambda_{m}\right)
\end{aligned}
$$

where

$$
v_{n}(t)=\frac{1}{n} \sum_{i=1}^{n} \delta_{i} t\left(T_{i}\right)-\langle t, \lambda\rangle_{n} .
$$

Let $S_{m}+S_{m^{\prime}}=\left\{t+t^{\prime}, t \in S_{m}, t^{\prime} \in S_{m^{\prime}}\right\}$. By Cauchy-Schwarz Inequality,

$$
\begin{aligned}
& \left.\left\|\widehat{\lambda}_{\widehat{m}}-\lambda_{m}\right\|_{n}^{2} \leq \operatorname{pen}(m)-\operatorname{pen}(\widehat{m})-2 \widehat{\left\langle\lambda_{\widehat{m}}\right.}-\lambda_{m}, \lambda_{m}-\lambda\right\rangle_{n} \\
& +2\left\|\widehat{\lambda}_{\widehat{m}}-\lambda_{m}\right\|_{\bar{F}_{T}} \sup _{t \in S_{m}+S_{\widehat{m}},\|t\|_{\bar{F}_{T}}=1} v_{n}(t)
\end{aligned}
$$

Moreover, for every numbers $b$ and $c, 2 b c \leq 2 b^{2}+(1 / 2) c^{2}$, so

$$
\begin{aligned}
\left\|\widehat{\lambda}_{\widehat{m}}-\lambda_{m}\right\|_{n}^{2} \leq & \operatorname{pen}(m)-\operatorname{pen}(\widehat{m})+2\left\|\widehat{\lambda}_{\widehat{m}}-\lambda_{m}\right\|_{n}\left\|\lambda_{m}-\lambda\right\|_{n}+\frac{1}{2}\left\|\widehat{\lambda}_{\widehat{m}}-\lambda_{m}\right\|_{\bar{F}_{T}} \\
& +2 \sup _{t \in S_{m}+S_{\widehat{m}},\|t\|_{\bar{F}_{T}}=1}\left(v_{n}(t)\right)^{2} .
\end{aligned}
$$

For every $p\left(m, m^{\prime}\right)$ function of $\left(m, m^{\prime}\right)$,

$$
\begin{aligned}
\left\|\widehat{\lambda}_{\widehat{m}}-\lambda_{m}\right\|_{n}^{2} \leq & \operatorname{pen}(m)-\operatorname{pen}(\widehat{m})+2 p(m, \widehat{m})+2\left\|\widehat{\lambda}_{\widehat{m}}-\lambda_{m}\right\|_{n}\left\|\lambda_{m}-\lambda\right\|_{n} \\
& \left.+\frac{1}{2} \| \widehat{\lambda}_{\widehat{m}}-\lambda_{m}\right) \|_{\bar{F}_{T}}^{2}+2 \sup _{t \in S_{m}+S_{\widehat{m}},\|t\|_{\bar{F}_{T}}=1}\left[\left(v_{n}(t)\right)^{2}-p(m, \widehat{m})\right] \\
\leq & \operatorname{pen}(m)-\operatorname{pen}(\widehat{m})+2 p(m, \widehat{m})+\frac{1}{4}\left\|\widehat{\lambda}_{\widehat{m}}-\lambda_{m}\right\|_{n}^{2}+4\left\|\lambda-\lambda_{m}\right\|_{n}^{2} \\
& +\frac{1}{2}\left\|\widehat{\lambda}_{\widehat{m}}-\lambda_{m}\right\|_{\bar{F}_{T}}^{2}+2 \sup _{t \in S_{m}+S_{\widehat{m}},\|t\|_{\bar{F}_{T}}=1}\left[\left(v_{n}(t)\right)^{2}-p(m, \widehat{m})\right]
\end{aligned}
$$

since $2 b c \leq 4 b^{2}+(1 / 4) c^{2}$ for every $b, c$. Thus

$$
\begin{gathered}
\frac{3}{4}\left\|\widehat{\lambda} \widehat{m}-\lambda_{m}\right\|_{n}^{2} \leq \\
+2 \operatorname{pen}_{t \in S_{m}+S_{\widehat{m}},\|t\|_{\bar{F}_{T}}=1}\left[\left(v_{n}(t)\right)^{2}-p(m, \widehat{m})\right] .
\end{gathered}
$$


On the set $\Delta_{1} \cap \Delta_{2}^{t h}=\Delta_{1}$ (see Proposition 4.1), $\left\|\widehat{\lambda}_{\widehat{m}}-\lambda_{m}\right\|_{n} \geq(3 / 4)\left\|\widehat{\lambda}_{\widehat{m}}-\lambda_{m}\right\|_{\bar{F}_{T}}$ hence

$$
\begin{aligned}
\left(\left(\frac{3}{4}\right)^{2}-\frac{1}{2}\right)\left\|\widehat{\lambda}_{\widehat{m}}-\lambda_{m}\right\|_{F_{T}}^{2} \leq & 4\left\|\lambda-\lambda_{m}\right\|_{n}^{2}+\operatorname{pen}(m)-\operatorname{pen}(\widehat{m})+2 p(m, \widehat{m}) \\
& +2 \sup _{t \in S_{m}+S_{\widehat{m}},\|t\|_{F_{T}}=1}\left[\left(v_{n}(t)\right)^{2}-p(m, \widehat{m})\right] .
\end{aligned}
$$

Moreover, we note that $\left\|\widehat{\lambda}_{\widehat{m}}-\lambda_{m}\right\|_{\bar{F}_{T}}^{2} \geq(1 / 2)\left\|\widehat{\lambda}_{\widehat{m}}-\lambda\right\|_{\bar{F}_{T}}^{2}-\left\|\lambda-\lambda_{m}\right\|_{\bar{F}_{T}}^{2}$ and $\mathbb{E}\left[\left\|\lambda-\lambda_{m}\right\|_{n}^{2}\right]=\left\|\lambda-\lambda_{m}\right\|_{F_{T}}^{2}$ so

$$
\begin{aligned}
\mathbb{E}\left[\left\|\widehat{\lambda}_{\widehat{m}}-\lambda\right\|_{\bar{F}_{T}}^{2} \mathbb{1}_{\Delta_{1}}\right] \leq & C_{2}\left\{\left\|\lambda-\lambda_{m}\right\|_{\bar{F}_{T}}^{2}+\mathbb{E}[\operatorname{pen}(m)-\operatorname{pen}(\widehat{m})+2 p(m, \widehat{m})]\right. \\
& \left.+\sup _{t \in S_{m}+S_{\widehat{m}},\|t\|_{\bar{F}_{T}}=1}\left[\left(v_{n}(t)\right)^{2}-p(m, \widehat{m})\right]\right\}
\end{aligned}
$$

where $C_{2}$ is a numerical constant.

On the one hand, let

$$
p_{1}\left(m, m^{\prime}\right)=\frac{B K^{2}}{2 \bar{F}_{0}} \frac{D_{m}+D_{m^{\prime}}}{n} \quad \text { and } \quad p_{2}\left(m, m^{\prime}\right)=\frac{B K^{2}\|\lambda\|_{\infty, A}}{2} \frac{D_{m}+D_{m^{\prime}}}{n}
$$

then

$$
\begin{aligned}
\operatorname{pen}(m)-\operatorname{pen}\left(\widehat{m}_{1}\right)+2 p_{1}\left(m, \widehat{m}_{1}\right) & =\frac{B \theta K^{2}}{\bar{F}_{0}} \frac{D_{m}}{n} \\
\text { and } \operatorname{pen}(m)-\operatorname{pen}\left(\widehat{m}_{2}\right)+2 p_{2}\left(m, \widehat{m}_{2}\right) & =B \theta K^{2}\|\lambda\|_{\infty, A} \frac{D_{m}}{n} .
\end{aligned}
$$

On the other hand, $v_{n}(t)$ is a centered process since

$$
\mathbb{E}\left[\delta_{i} t\left(T_{i}\right)\right]=\int_{A} t(x) \lambda(x) \bar{F}_{T}(x) d x=\mathbb{E}\left[\langle t, \lambda\rangle_{n}\right]
$$

(see Eq. 7). Therefore, we insert the mean term $\int_{A} t(x) \lambda(x) \bar{F}_{T}(x) d x$ to obtain the sum of two variance-type terms. More precisely, we define

$$
\begin{aligned}
& v_{n, 1}(t)=\frac{1}{n} \sum_{i=1}^{n} \delta_{i} t\left(T_{i}\right)-\int_{A} t(x) \lambda(x) \bar{F}_{T}(x) d x \quad \text { and } \\
& v_{n, 2}(t)=\frac{1}{n} \sum_{i=1}^{n} \int_{A} t(x) \lambda(x) \mathbb{1}_{T_{i} \geq x} d x-\int_{A} t(x) \lambda(x) \bar{F}_{T}(x) d x .
\end{aligned}
$$


Then, as $(b+c)^{2} \leq \frac{3}{2} b^{2}+3 c^{2}$ for every $b$ and $c$,

$$
\begin{aligned}
& \mathbb{E}\left[\sup _{t \in S_{m}+S_{\widehat{m}},\|t\|_{\bar{F}_{T}}=1}\left(\left(v_{n}(t)\right)^{2}-p(m, \widehat{m})\right)\right] \\
& \leq \frac{3}{2} \mathbb{E}\left[\sup _{t \in S_{m}+S_{\widehat{m}},\|t\|_{\bar{F}_{T}}=1}\left(\left(v_{n, 1}(t)\right)^{2}-\frac{2}{3} p(m, \widehat{m})\right)_{+}\right] \\
& +3 \mathbb{E}\left[\sup _{t \in S_{m}+S_{\widehat{m}},\|t\|_{\bar{F}_{T}}=1}\left(v_{n, 2}(t)\right)^{2}\right] .
\end{aligned}
$$

Moreover, the two terms above are upper-bounded as follows.

\section{Lemma 6.1}

$$
\mathbb{E}\left[\sup _{t \in S_{m}+S_{\widehat{m}},\|t\|_{\bar{F}_{T}}=1}\left(v_{n, 2}(t)\right)^{2}\right] \leq \frac{1}{\bar{F}_{0} n}\|\lambda\|_{\bar{F}_{T}}^{2} .
$$

Lemma 6.2 Under $\mathbf{A}_{\text {mod }}^{(\mathbf{j})}$

$$
\mathbb{E}\left[\left(\sup _{t \in S_{m}+S_{\widehat{m}_{j}},\|t\|_{\bar{F}_{T}}=1}\left(v_{n, 1}(t)\right)^{2}-\frac{2}{3} p_{j}\left(m, \widehat{m}_{j}\right)\right)_{+}\right] \leq \frac{C^{\prime}}{n}
$$

for some constant $C^{\prime}$ depending on $\left(K,\|\lambda\|_{\infty, A}, \bar{F}_{0}\right)$.

Finally Eqs. 18, 20, 21 and Lemmas 6.1 and 6.2 provide Eq. 16, which ends the proof of Proposition 6.1.

Proof of Lemma 6.1 For every $m, \widehat{m} \in J_{n}, S_{m}+S_{\widehat{m}} \subset S_{n}$, hence

$$
\mathbb{E}\left[\sup _{t \in S_{m}+S_{\widehat{m}},\|t\|_{\bar{F}_{T}}=1}\left(v_{n, 2}(t)\right)^{2}\right] \leq \mathbb{E}\left[\sup _{t \in S_{n},\|t\|_{\bar{F}_{T}}=1}\left(v_{n, 2}(t)\right)^{2}\right] .
$$

Besides,

$$
\left\{t,\|t\|_{F_{T}}^{2} \leq 1\right\} \subset\left\{t,\|t\|^{2} \leq \bar{F}_{0}^{-1}\right\}
$$


so

$$
\begin{aligned}
\mathbb{E}\left[\sup _{t \in S_{m}+S_{\widehat{m}_{i}},\|t\|_{\bar{F}_{T}}=1}\left(v_{n, 2}(t)\right)^{2}\right] & \leq \mathbb{E}\left[\sup _{t \in S_{n},\|t\|^{2} \leq 1 / \bar{F}_{0}}\left(v_{n, 2}(t)\right)^{2}\right] \\
& =\mathbb{E}\left[\sup _{\sum_{k=1}^{N_{n}} a_{k}^{2} \leq 1 / \bar{F}_{0}}\left(\sum_{k=1}^{N_{n}} a_{k}\left(\left\langle\phi_{k}^{n}, \lambda\right\rangle_{n}-\left\langle\phi_{k}^{n}, \lambda\right\rangle_{\bar{F}_{T}}\right)\right)^{2}\right]
\end{aligned}
$$

where $\left(\phi_{1}^{n}, \ldots, \phi_{N_{n}}^{n}\right)$ is an $\|\cdot\|$-orthonormal basis of $S_{n}$. With Cauchy-Schwarz Inequality, we obtain

$$
\begin{aligned}
& \mathbb{E}\left[\sup _{\left.t \in S_{m}+S_{\widehat{m},\|t\|_{\bar{F}_{T}}=1}\left(v_{n, 2}(t)\right)^{2}\right]} \leq \frac{1}{\bar{F}_{0}} \sum_{k=1}^{N_{n}} \mathbb{E}\left[\left(\left\langle\phi_{k}^{n}, \lambda\right\rangle_{n}-\left\langle\phi_{k}^{n}, \lambda\right\rangle_{\bar{F}_{T}}\right)^{2}\right]\right. \\
&=\frac{1}{\bar{F}_{0}} \sum_{k=1}^{N_{n}} \frac{1}{n} \operatorname{Var}\left[\int_{A} \lambda(x) \phi_{k}^{n}(x) \mathbb{1}_{\left\{T_{1} \geq x\right\}} d x\right] \\
& \leq \frac{1}{\bar{F}_{0} n} \mathbb{E}\left[\sum_{k=1}^{N_{n}}\left\langle\phi_{k}^{n}, \lambda(.) \mathbb{1}_{\left\{T_{1} \geq .\right\}}\right\rangle^{2}\right] \\
&=\frac{1}{\bar{F}_{0} n} \mathbb{E}\left[\left\|\left(\lambda(.) \mathbb{1}_{\left\{T_{1} \geq .\right\}}\right)_{S_{n}}\right\|^{2}\right]
\end{aligned}
$$

where $\left(\lambda(.) \mathbb{1}_{\left\{T_{1} \geq .\right\}}\right) S_{n}$ denotes the $L^{2}$-orthogonal projection of $\lambda(.) \mathbb{1}_{\left\{T_{1} \geq .\right\}}$ on $S_{n}$. Thus

$$
\mathbb{E}\left[\sup _{t \in S_{m}+S_{\widehat{m}},\|t\|_{\bar{F}_{T}}=1}\left(v_{n, 2}(t)\right)^{2}\right] \leq \frac{1}{\bar{F}_{0} n} \mathbb{E}\left[\left\|\lambda(.) \mathbb{1}_{\left\{T_{1} \geq .\right\}}\right\|^{2}\right]=\frac{1}{\bar{F}_{0} n}\|\lambda\|_{\bar{F}_{T}}^{2} .
$$

Proof of Lemma 6.2 For $j=1$ or 2, we have

$$
\begin{aligned}
& \mathbb{E}\left[\left(\sup _{\left.\left.t \in S_{m}+S_{\widehat{m}_{j},\|t\|_{\bar{F}_{T}}=1}\left(v_{n, 1}(t)\right)^{2}-\frac{2}{3} p_{j}\left(m, \widehat{m}_{j}\right)\right)_{+}\right]}\right.\right. \\
& \leq \sum_{m^{\prime} \in J_{n}} \mathbb{E}\left[\left(\sup _{t \in S_{m}+S_{m^{\prime}},\|t\|_{\bar{F}_{T}}=1}\left(v_{n, 1}(t)\right)^{2}-\frac{2}{3} p_{j}\left(m, m^{\prime}\right)\right)_{+}\right] .
\end{aligned}
$$

Besides, for every models $S_{m}, S_{m^{\prime}}$, we upper bound the term $\mathbb{E}\left[\left(\sup _{t \in S_{m}+S_{m^{\prime}},\|t\|_{\bar{F}_{T}}=1}\left(v_{n, 1}(t)\right)^{2}-p_{j}\left(m, m^{\prime}\right)\right)_{+}\right]$with Talagrand Inequality (Theorem 4$)$. 
- Consider $j=1$. Under $\mathbf{A}_{\text {mod }}^{(\mathbf{1})}, S_{m} \subset S_{m^{\prime}}$ or $S_{m^{\prime}} \subset S_{m}$. Thus $S_{m}+S_{m^{\prime}}$ is equal either to $S_{m}$ or to $S_{m^{\prime}}$. Let $D_{m+m^{\prime}}=\max \left(D_{m}, D_{m^{\prime}}\right)$ denote the dimension of $S_{m}+S_{m^{\prime}}$, and $\left(\phi_{1}^{m+m^{\prime}}, \ldots, \phi_{D_{m+m^{\prime}}}^{m+m^{\prime}}\right)$ be the orthonormal basis of $S_{m}+S_{m^{\prime}}$ defined as $\phi_{k}^{m+m^{\prime}}=\phi_{k}^{m}$ if $S_{m}+S_{m^{\prime}}=S_{m}$, and $\phi_{k}^{m^{\prime}}$ if $S_{m}+S_{m^{\prime}}=S_{m^{\prime}}$.

Let us compute the term $\mathbb{H}$. With Eq. 22,

$$
\begin{aligned}
\mathbb{E}\left[\sup _{t \in S_{m}+S_{m^{\prime}},\|t\|_{\bar{F}_{T}}=1}\left(v_{n, 1}(t)\right)^{2}\right] & \left.\leq \mathbb{E}\left[\sup _{t \in S_{m}+S_{m^{\prime}},\|t\| \leq 1 / \bar{F}_{0}}\left(v_{n, 1}(t)\right)^{2}\right)_{+}\right] \\
& \leq \frac{1}{\bar{F}_{0}} \sum_{k=1}^{D_{m+m^{\prime}}} \frac{1}{n} \operatorname{Var}\left[\delta_{1} \phi_{k}^{m+m^{\prime}}\left(T_{1}\right)\right] \\
& \leq \frac{1}{\bar{F}_{0}} \sum_{k=1}^{D_{m+m^{\prime}}} \frac{1}{n} \mathbb{E}\left[\left(\phi_{k}^{m+m^{\prime}}\right)^{2}\left(T_{1}\right)\right] .
\end{aligned}
$$

Besides, according to Eq. 5,

$$
\begin{aligned}
\mathbb{E}\left[\sup _{t \in S_{m}+S_{m^{\prime}},\|t\|_{\bar{F}_{T}}=1}\left(v_{n, 1}(t)\right)^{2}\right] & \leq \frac{1}{n \bar{F}_{0}} \sup _{x \in A}\left|\sum_{k=1}^{D_{m+m^{\prime}}}\left(\phi_{k}^{m+m^{\prime}}(x)\right)^{2}\right| \\
& \leq \frac{K^{2}\left(D_{m}+D_{m^{\prime}}\right)}{\bar{F}_{0} n}=\mathbb{H}^{2}
\end{aligned}
$$

Then, with $B>3,(2 / 3) p_{1}\left(m, m^{\prime}\right)=\theta \mathbb{H}^{2}$ for some $\theta>1$. Let us compute the terms $c$ and $v$.

$$
\begin{aligned}
\sup _{t \in S_{m}+S_{m^{\prime}},\|t\|_{F_{T}}=1}\left\|\delta_{1} t\left(T_{1}\right)\right\|_{\infty} \leq & \sup _{t \in S_{m}+S_{m^{\prime}},\|t\|^{2} \leq 1 / \bar{F}_{0}}\left(\sup _{x \in A}|t(x)|\right) \\
= & \sup _{\sum_{k=1}^{D_{m+m^{\prime}}} a_{k}^{2} \leq 1 / \bar{F}_{0}}\left(\sup _{x \in A}\left|\sum_{k=1}^{D_{m+m^{\prime}}} a_{k} \phi_{k}^{m+m^{\prime}}(x)\right|\right)
\end{aligned}
$$

With Cauchy-Schwarz Inequality,

$$
\begin{aligned}
\sup _{t \in S_{m}+S_{m^{\prime}},\|t\|_{F_{T}}=1}\left\|\delta_{1} t\left(T_{1}\right)\right\|_{\infty} & \leq \frac{1}{\sqrt{\bar{F}_{0}}} \sup _{x \in A}\left|\sum_{k=1}^{D_{m+m^{\prime}}}\left(\phi_{k}^{m+m^{\prime}}(x)\right)^{2}\right| \\
& \leq \frac{K}{\sqrt{\bar{F}_{0}}} \sqrt{D_{m}+D_{m^{\prime}}}=b .
\end{aligned}
$$


Moreover, Eq. 7 entails

$$
\begin{aligned}
\sup _{t \in S_{m}+S_{m^{\prime}},\|t\|_{\bar{F}_{T}}=1} \operatorname{Var}\left(\delta_{1} t\left(T_{1}\right)\right) & \leq \sup _{t \in S_{m}+S_{m^{\prime}},\|t\|_{\bar{F}_{T}}=1} \mathbb{E}\left[\delta_{1} t^{2}\left(T_{1}\right)\right] \\
& =\sup _{t \in S_{m}+S_{m^{\prime}},\|t\|_{\bar{F}_{T}}=1} \int_{A} t^{2}(x) \lambda(x) \bar{F}_{T}(x) d x \\
& \leq\|\lambda\|_{\infty, A}=v .
\end{aligned}
$$

Then Talagrand Inequality leads to

$$
\begin{aligned}
& \mathbb{E}\left[\left(\sup _{t \in S_{m}+S_{m^{\prime}},\|t\|_{\bar{F}_{T}}=1}\left(v_{n, 1}(t)\right)^{2}-\frac{2}{3} p_{1}\left(m, m^{\prime}\right)\right)_{+}\right] \\
& \leq \bar{C} \frac{\|\lambda\|_{\infty, A}}{n} \exp \left(-\kappa \frac{K^{2}\left(D_{m}+D_{m^{\prime}}\right)}{\bar{F}_{0}\|\lambda\|_{\infty, A}}\right)+\bar{C}^{\prime} \frac{K^{2}\left(D_{m}+D_{m^{\prime}}\right)}{\bar{F}_{0}^{2} n^{2}} \exp \left(-\kappa^{\prime} \sqrt{n}\right) .
\end{aligned}
$$

Thus, with Eq. 6,

$$
\sum_{m^{\prime} \in \mathcal{M}_{n}} \mathbb{E}\left[\left(\sup _{t \in S_{m}+S_{m^{\prime}},\|t\|_{\bar{F}_{T}}=1}\left(v_{n, 1}(t)\right)^{2}-\frac{2}{3} p_{1}\left(m, m^{\prime}\right)\right)_{+}\right] \leq \frac{C^{\prime}}{n}
$$

which concludes the proof of Lemma 6.2 for $j=1$.

- Consider $j=2$. Let $\left(\psi_{1}, \ldots, \psi_{D_{m+m^{\prime}}}\right)$ be a $\|\cdot\|_{F_{T}}$-orthonormal basis of $S_{m}+S_{m^{\prime}}$. Similarly to Eq. 23,

$$
\begin{aligned}
\mathbb{E}\left[\sup _{t \in S_{m}+S_{m^{\prime}},\|t\|_{F_{T}}=1}\left(v_{n, 1}(t)\right)^{2}\right] & \leq \frac{1}{n} \sum_{k=1}^{D_{m+m^{\prime}}} \operatorname{Var}\left(\delta_{1} \psi_{k}^{2}\left(X_{1}\right)\right) \leq \frac{1}{n} \sum_{k=1}^{D_{m+m^{\prime}}} \mathbb{E}\left[\delta_{1} \psi_{k}^{2}\left(X_{1}\right)\right] \\
& =\frac{1}{n} \sum_{k=1}^{D_{m+m^{\prime}}} \int_{A} \psi_{k}^{2}(x) \lambda(x) \bar{F}_{T}(x) d x \\
& \leq\|\lambda\|_{\infty, A} \frac{D_{m}+D_{m^{\prime}}}{n}=\mathbb{H}^{2}
\end{aligned}
$$

Besides, according to $\mathbf{A}_{\text {mod }}^{(\mathbf{2})}$,

$$
\sup _{t \in S_{m}+S_{m^{\prime}},\|t\|_{\bar{F}_{T}}=1}\left\|\delta_{1} t\left(T_{1}\right)\right\|_{\infty} \leq \sup _{t \in S_{m}+S_{m^{\prime}},\|t\|^{2} \leq 1 / \bar{F}_{0}} \sup _{x \in A}|t(x)| \leq \frac{K}{\sqrt{\bar{F}_{0}}} \sqrt{N_{n}}=b
$$

and the end of the proof is similar to the case $j=1$. 


\subsection{Proof of Proposition 6.2}

Let $m \leq N_{n}$,

$$
\left\|\widehat{\lambda}_{m}-\lambda\right\|_{\bar{F}_{T}} \leq\left\|\widehat{\lambda}_{m}\right\|_{\bar{F}_{T}}+\|\lambda\|_{\bar{F}_{T}} \leq\left\|\widehat{\lambda}_{m}\right\|+\|\lambda\|_{\bar{F}_{T}} .
$$

On $\left(\Delta_{2}^{t h}\right)^{c},\left\|\widehat{A}_{m}\right\|=0$. On $\Delta_{2}^{t h}$,

$$
\left\|\widehat{A}_{m}\right\| \leq \max \left(\operatorname{Sp}\left(\widehat{G}_{m}^{-1}\right)\right)\left\|\widehat{V}_{m}\right\|=\left[\min \left(\operatorname{Sp}\left(\widehat{G}_{m}\right)\right)\right]^{-1}\left\|\widehat{V}_{m}\right\| \leq \frac{4}{3 \bar{F}_{0}}
$$

Hence

$$
\begin{aligned}
\left\|\widehat{\lambda}_{m}-\lambda\right\|_{\bar{F}_{T}} & \leq \frac{4}{3 \bar{F}_{0}}\left[\sum_{k=1}^{D_{m}}\left(\frac{1}{n} \sum_{i=1}^{n} \phi_{k}^{m}\left(T_{i}\right) \delta_{i}\right)^{2}\right]^{1 / 2}+\|\lambda\|_{\bar{F}_{T}} \\
& \leq \frac{4}{3 \bar{F}_{0}}\left[\sum_{k=1}^{D_{m}} \frac{1}{n} \sum_{i=1}^{n}\left(\phi_{k}^{m}\right)^{2}\left(T_{i}\right)\right]^{1 / 2}+\|\lambda\|_{\bar{F}_{T}} \\
& \leq \frac{4}{3 \bar{F}_{0}} \sup _{x \in A}\left|\sum_{k=1}^{D_{m}}\left(\phi_{k}^{m}(x)\right)^{2}\right|^{1 / 2}+\|\lambda\|_{\bar{F}_{T}} \\
& \leq \frac{4 K{ }_{N_{n}}}{3 \bar{F}_{0}}+\|\lambda\|_{\bar{F}_{T} .}
\end{aligned}
$$

\subsection{Proof of Proposition 6.3}

The proof of Proposition 6.3 is inspired from Baraud (2002). From the definition of $\Delta_{1}$,

$$
\Delta_{1}^{c}=\left\{\left|\|t\|_{n}^{2}-\|t\|_{\bar{F}_{T}}^{2}\right|>\frac{1}{4}\|t\|_{\bar{F}_{T}}^{2}, \forall t \in S_{n}\right\}=\left\{\sup _{t \in S_{n},\|t\|_{\bar{F}_{T}}=1} \eta_{n}\left(t^{2}\right)>\frac{1}{4}\right\}
$$

where $\eta_{n}(t)=\frac{1}{n} \sum_{i=1}^{n}\left(\int_{A} t(x) \mathbb{1}_{\left\{T_{i} \geq x\right\}} d x-\int_{A} t(x) \bar{F}_{T}(x) d x\right)$. Let $\left(\psi_{1}, \ldots, \psi_{N_{n}}\right)$ be an orthonormal base of the global space $S_{n}$ for the norm $\|\cdot\|_{F_{T}}$, then

$$
\Delta_{1}^{c}=\left\{\sup _{\sum a_{k}^{2}=1} \sum_{k, k^{\prime}=1}^{N_{n}} a_{k} a_{k^{\prime}} S_{k, k^{\prime}}>\frac{1}{4}\right\}
$$

where

$$
S_{k, k^{\prime}}=\frac{1}{n} \sum_{i=1}^{n}\left(\int_{A} \psi_{k}(x) \psi_{k^{\prime}}(x) \mathbb{1}_{\left\{T_{i} \geq x\right\}} d x-\int_{A} \psi_{k}(x) \psi_{k^{\prime}}(x) \bar{F}_{T}(x) d x\right) .
$$


On the one hand, let $k, k^{\prime}$ be fixed. Let

$\frac{1}{n} \sum_{i=1}^{n} \mathbb{E}\left[\left(\int_{A} \psi_{k}(x) \psi_{k^{\prime}}(x) \mathbb{1}_{\left\{T_{i} \geq x\right\}} d x\right)^{2}\right]=\mathbb{E}\left[\left(\int_{A} \psi_{k}(x) \psi_{k^{\prime}}(x) \mathbb{1}_{\left\{T_{1} \geq x\right\}} d x\right)^{2}\right]=v_{k, k^{\prime}}$

and for every $l \geq 2$ :

$\mathbb{E}\left[\left(\int_{A} \psi_{k}(x) \psi_{k^{\prime}}(x) \mathbb{1}_{\left\{T_{1} \geq x\right\}} d x\right)_{+}^{l}\right] \leq \mathbb{E}\left[\left(\int_{A} \psi_{k}(x) \psi_{k^{\prime}}(x) \mathbb{1}_{\left\{T_{1} \geq x\right\}} d x\right)^{2}\right.$
$\left.\times\left(\int_{A}\left|\psi_{k}(x) \psi_{k^{\prime}}(x)\right| d x\right)^{l-2}\right]$
$\leq v_{k, k^{\prime}}\left(\int_{A} \psi_{k}^{2}(x) d x \int_{A} \psi_{k^{\prime}}^{2}(x) d x\right)^{(l / 2)-1}$
$\leq v_{k, k^{\prime}}\left(\frac{1}{\bar{F}_{0}}\right)^{l-2}$
$\times\left(\int_{A} \psi_{k}^{2}(x) \bar{F}_{T}(x) d x \int_{A} \psi_{k^{\prime}}^{2}(x) \bar{F}_{T}(x) d x\right)^{(l / 2)-1}$
$=v_{k, k^{\prime}}\left(\frac{1}{\bar{F}_{0}}\right)^{l-2}=v_{k, k^{\prime}} c_{k, k^{\prime}}^{l-2}$

with $c_{k, k^{\prime}}=1 / \bar{F}_{0}$. Thus Bernstein inequality (Theorem 3 ) provides the following upper bound.

$$
P\left[\left|S_{k, k^{\prime}}\right| \geq \sqrt{2 v_{k, k^{\prime}} x}+c_{k, k^{\prime}} x\right] \leq 2 \exp (-n x)
$$

On the other hand,

$$
\begin{aligned}
& \left\{\left|S_{k, k^{\prime}}\right|<\sqrt{2 v_{k, k^{\prime}} x}+c_{k, k^{\prime}} x, \forall k, k^{\prime}=1, \ldots N_{n}\right\} \\
& \subset\left\{\sum_{k, k^{\prime}=1}^{N_{n}}\left|a_{k}\right|\left|S_{k, k^{\prime}}\right|\left|a_{k^{\prime}}\right|<\sqrt{2 x} \sum_{k, k^{\prime}=1}^{N_{n}}\left|a_{k}\right| \sqrt{v_{k, k^{\prime}}}\left|a_{k^{\prime}}\right|\right. \\
& \left.\quad+x \sum_{k, k^{\prime}=1}^{N_{n}}\left|a_{k}\right| c_{k, k^{\prime}}\left|a_{k^{\prime}}\right|, \forall\left(a_{k}\right)_{k=1, \ldots N_{n}}\right\}
\end{aligned}
$$




$$
\begin{aligned}
& \subset\left\{\sup _{\sum a_{k}^{2}=1} \sum_{k, k^{\prime}=1}^{N_{n}}\left|a_{k}\right|\left|S_{k, k^{\prime}}\right|\left|a_{k^{\prime}}\right|<\sqrt{2 x} \sup _{\sum a_{k}^{2}=1} \sum_{k, k^{\prime}=1}^{N_{n}}\left|a_{k}\right| \sqrt{v_{k, k^{\prime}}}\left|a_{k^{\prime}}\right|\right. \\
& \left.+x \sup _{\sum a_{k}^{2}=1} \sum_{k, k^{\prime}=1}^{N_{n}}\left|a_{k}\right| c_{k, k^{\prime}}\left|a_{k^{\prime}}\right|\right\} \\
& =\left\{\sup _{t \in S_{n},\|t\|_{F_{T}} \leq 1}\left|\eta_{n}\left(t^{2}\right)\right| \leq \sqrt{2 x} \rho(V)+x \rho(C)\right\}
\end{aligned}
$$

where $\rho(M)$ denotes the maximum of the spectrum of $M$, and $V$ and $C$ denote the following matrix.

$$
V=\left(\sqrt{v_{k, k^{\prime}}}\right)_{k, k^{\prime}=1, \ldots, N_{n}} \quad \text { and } \quad C=\left(c_{k, k^{\prime}}\right)_{k, k^{\prime}=1, \ldots, N_{n}}
$$

Thus for every $x \geq 0$,

$$
\begin{aligned}
& P\left[\sup _{t \in S_{n},\|t\|_{\bar{F}_{T}} \leq 1}\left|\eta_{n}\left(t^{2}\right)\right|>\sqrt{2 x} \rho(V)+x \rho(C)\right] \\
& \leq \sum_{k, k^{\prime}} P\left[\left|S_{k, k^{\prime}}\right|>\sqrt{2 v_{k, k^{\prime}} x}+c_{k, k^{\prime}} x\right] \leq 2 N_{n}^{2} \exp (-n x) .
\end{aligned}
$$

In order to upper bound $P\left[\Delta_{1}^{c}\right]$, we choose $x$ such that $\sqrt{2 x} \rho(V) \leq 1 / 8$ and $x \rho(C) \leq$ $1 / 8$. Let $L(\psi)=\max \left(\rho(C), 16 \rho(V)^{2}\right)$ then,

$$
P\left[\Delta_{1}^{c}\right] \leq 2 \exp \left(-\frac{n}{8 L(\psi)}\right) .
$$

Let upper bound $L(\psi)$. Applying two times Cauchy-Schwarz inequality, we obtain

$$
\begin{aligned}
(\rho(V))^{2} & =\sup _{\sum a_{k}^{2}=1}\left[\sum_{k=1}^{N_{n}}\left|a_{k}\right|\left(\sum_{k^{\prime}=1}^{N_{n}}\left|a_{k^{\prime}}\right| \sqrt{v_{k, k^{\prime}}}\right)\right]^{2} \\
& \leq \sup _{\sum a_{k}^{2}=1}\left(\sum_{k=1}^{N_{n}} a_{k}^{2}\right)\left(\sum_{k=1}^{N_{n}}\left[\sum_{k^{\prime}=1}^{N_{n}}|| a_{k^{\prime}} \mid \sqrt{v_{k, k^{\prime}}}\right]^{2}\right) \\
& =\sup _{\sum a_{k}^{2}=1} \sum_{k=1}^{N_{n}}\left(\sum_{k^{\prime}=1}^{N_{n}}\left|a_{k^{\prime}}\right| \sqrt{v_{k, k^{\prime}}}\right)^{2} \leq \sum_{k=1}^{N_{n}}\left(\sum_{k^{\prime}=1}^{N_{n}} v_{k, k^{\prime}}\right) .
\end{aligned}
$$


We replace $v_{k, k^{\prime}}$ by its expression.

$$
\begin{aligned}
(\rho(V))^{2} & \leq \sum_{k=1}^{N_{n}} \mathbb{E}\left[\sum_{k^{\prime}=1}^{N_{n}}\left\langle\psi_{k^{\prime}}, \psi_{k} \mathbb{1}_{\left\{T_{1} \geq .\right\}}\right\rangle^{2}\right] \\
& \leq \frac{1}{\bar{F}_{0}} \sum_{k=1}^{N_{n}} \mathbb{E}\left[\sum_{k^{\prime}=1}^{N_{n}}\left\langle\psi_{k^{\prime}}, \psi_{k} \mathbb{1}_{\left\{T_{1} \geq .\right\}}\right\rangle_{F_{T}}\right]
\end{aligned}
$$

Besides, $\sqrt{\sum_{k^{\prime}=1}^{N_{n}}\left\langle\psi_{k^{\prime}}, \psi_{k} \mathbb{1}_{\left\{T_{1} \geq .\right\}}\right\rangle_{F_{T}}^{2}}$ is equal to the norm of the $\|\cdot\|_{\bar{F}_{T}}$-projection of $\psi_{k} \mathbb{1}_{\left\{T_{1} \geq .\right\}}$ on $S_{n}$, so

$$
\sum_{k^{\prime}=1}^{N_{n}}\left\langle\psi_{k^{\prime}}, \psi_{k} \mathbb{1}_{\left\{T_{1} \geq .\right\}}\right\rangle_{\bar{F}_{T}} \leq\left\|\psi_{k} \mathbb{1}_{\left\{T_{1} \geq .\right\}}\right\|_{\bar{F}_{T}}^{2} \leq\left\|\psi_{k}\right\|_{\bar{F}_{T}}^{2}=1 .
$$

Hence $(\rho(V))^{2} \leq N_{n} / \bar{F}_{0}^{2}$.

Besides,

$$
\begin{aligned}
\rho(C) & =\frac{1}{\bar{F}_{0}} \sup _{\sum a_{k}^{2}=1}\left(\sum_{k, k^{\prime}=1}^{N_{n}}\left|a_{k}\right|\left|a_{k^{\prime}}\right|\right) \\
& \leq \frac{1}{\bar{F}_{0}} \sup _{\sum a_{k}^{2}=1} \sqrt{\sum_{k=1}^{N_{n}} a_{k}^{2}} \sqrt{\sum_{k=1}^{N_{n}}\left(\sum_{k^{\prime}=1}^{N_{n}}\left|a_{k^{\prime}}\right|\right)^{2}} \\
& \leq \frac{1}{\bar{F}_{0}} \sup _{\sum a_{k}^{2}=1} \sqrt{N_{n}} \sum_{k^{\prime}=1}^{N_{n}}\left|a_{k^{\prime}}\right| \leq \frac{N_{n}}{\bar{F}_{0}} .
\end{aligned}
$$

Finally $L(\psi) \leq \max \left(N_{n} / \bar{F}_{0}, 16 N_{n} / \bar{F}_{0}^{2}\right)=16 N_{n} / \bar{F}_{0}^{2}$ and

$$
P\left[\Delta_{1}^{c}\right] \leq \exp \left(-C_{2} \bar{F}_{0}^{2} \frac{n}{N_{n}}\right) .
$$

6.5 Comment about the constant in the penalty

Provided that the set $\Delta_{2}^{t h}$ is replaced by

$$
\left(\Delta_{2}^{t h}\right)^{\prime}=\left\{\min \left(\operatorname{Sp}\left(\widehat{G}_{m}\right) \geq(1-\alpha) \bar{F}_{0}\right\},\right.
$$

and the inequalities of the kind $2 b c \leq 2 b^{2}+(1 / 2) c^{2}$ by $2 b c \leq(1 / \beta) b^{2}+\beta c^{2}$ with $\alpha, \beta$ small enough, Theorem 1 holds for any constant $B>1$. 


\section{Proof of Theorem 2}

The following Propositions are intermediate results to prove Theorem 2. Assume that A frame holds.

Proposition 7.1 1. Under $\mathbf{A}_{\text {mod' }}^{(\mathbf{1})}$

$$
\mathbb{E}\left[\left\|\widehat{\lambda}_{\widehat{m}_{1}}-\lambda\right\|_{\bar{F}_{T}}^{2} \mathbb{1}_{\Delta_{1} \cap \Delta_{2} \cap \Delta_{3}}\right] \leq C \inf _{m \in \mathcal{M}_{n}}\left[\inf _{t \in S_{m}}\|t-\lambda\|_{\bar{F}_{T}}^{2}+\operatorname{pen}_{1}^{t h}(m)\right]+\frac{C^{\prime}}{n}
$$

where $C$ is a numerical constant and $C^{\prime}$ depends on $\left(K, \bar{F}_{0},\|\lambda\|_{\infty}\right)$.

2. Under $\mathbf{A}_{\text {mod }}^{(2)}$

$$
\mathbb{E}\left[\left\|\widehat{\lambda}_{\widehat{m}_{2}}-\lambda\right\|_{\bar{F}_{T}}^{2} \mathbb{1}_{\Delta_{1} \cap \Delta_{2} \cap \Delta_{3} \cap \Delta_{4}}\right] \leq C \inf _{m \in \mathcal{M}_{n}}\left[\inf _{t \in S_{m}}\|t-\lambda\|_{F_{T}}^{2}+\operatorname{pen}_{2}^{t h}(m)\right]+\frac{C^{\prime}}{n}
$$

where $C$ is a numerical constant and $C^{\prime}$ depends on $\left(K, \bar{F}_{0},\|\lambda\|_{\infty}\right)$.

Proposition 7.2 There exists a numerical constant $C_{2}$ such that, provided that $\alpha_{n} \leq$ $\bar{F}_{0} / 2$,

$$
P\left[\Delta_{3}^{c}\right] \leq 2 \exp \left(-C_{2} n \bar{F}_{0}\right)
$$

Proposition 7.3 Assume that Eq. 14 is satisfied, then

$$
P\left[\Delta_{4}^{c} \cap \Delta_{1}\right] \leq 4 D \exp \left(-C \frac{n}{D}\right)
$$

where $C$ depends on $\left(\nu, \bar{F}_{0},\|\lambda\|_{\infty}, a\right)$.

\subsection{Proof of Theorem 2}

For every model $m$, similarly to Proposition 6.2,

$$
\left\|\widehat{\lambda}_{m}-\lambda\right\|_{\bar{F}_{T}} \leq \frac{5 K \sqrt{N_{n}}}{3 \widehat{F}_{0}}+\|\lambda\|_{\bar{F}_{T}} \leq \frac{5 K \sqrt{N_{n}}}{3 \alpha_{n}}+\|\lambda\|_{\bar{F}_{T}}=\frac{5}{3} K n+\|\lambda\|_{\bar{F}_{T}}
$$

since $\widehat{F}_{0} \geq \alpha_{n}$ and $N_{n} \leq n$.

1. Let $j=1$.

$$
\mathbb{E}\left[\left\|\widehat{\lambda}_{\widehat{m}_{1}}-\lambda\right\|_{\bar{F}_{T}}^{2} \mathbb{1}_{\left(\Delta_{1} \cap \Delta_{2} \cap \Delta_{3}\right)^{c}}\right] \leq\left(\frac{5}{3} K n+\|\lambda\|_{\bar{F}_{T}}\right)^{2} P\left[\left(\Delta_{1} \cap \Delta_{2} \cap \Delta_{3}\right)^{c}\right] .
$$


Besides, according to Proposition 4.1, $\Delta_{1} \cap \Delta_{2} \cap \Delta_{3}=\Delta_{1} \cap \Delta_{3}$. Therefore, Propositions 6.3 and 7.2 entail

$$
\begin{aligned}
P\left[\left(\Delta_{1} \cap \Delta_{2} \cap \Delta_{3}\right)^{c}\right] & \leq P\left[\left(\Delta_{1}^{c} \cap \Delta_{3}\right) \cup\left(\Delta_{2}^{c} \cap \Delta_{3}\right)\right]+P\left[\Delta_{3}^{c}\right] \\
& \leq 2 \exp \left(-C_{1} \bar{F}_{0} n\right)+\exp \left(-C_{2} \bar{F}_{0} \frac{n}{N_{n}}\right) .
\end{aligned}
$$

Thus similarly to Eq. 17,

$$
\mathbb{E}\left[\left\|\widehat{\lambda}_{m_{1}}-\lambda\right\|_{F_{T}}^{2} \mathbb{1}_{\left(\Delta_{1} \cap \Delta_{2} \cap \Delta_{3}\right)^{c}}\right] \leq \frac{C^{\prime}}{n} .
$$

Proposition 7.1 and Eq. 25 conclude the proof of Theorem 2 for $j=1$.

2. Let $j=2$.

$$
\begin{aligned}
P\left[\left(\Delta_{1} \cap \Delta_{2} \cap \Delta_{3} \cap \Delta_{4}\right)^{c}\right] & =P\left[\left(\Delta_{1}^{c} \cup \Delta_{3}^{c} \cup \Delta_{4}^{c}\right) \cap \Delta_{1}\right]+P\left[\Delta_{1}^{c}\right] \\
& \leq P\left[\Delta_{1}^{c}\right]+P\left[\Delta_{3}^{c}\right]+P\left[\Delta_{4}^{c} \cap \Delta_{1}\right]
\end{aligned}
$$

and Propositions 6.3, 7.2 and 7.3 allow to conclude similarly to the case $j=1$.

\subsection{Proof of Proposition 7.1}

We only expose the proof of (1) since the proof of (2) is very similar. The proof of Proposition 7.1 follows the same line as Proposition 6.1, let us point out the slight differences. Equations 18 and 21, as well as Lemma 6.1 hold. Hence, for every model $m$ and every $\lambda \in S_{m}$,

$$
\begin{aligned}
& \mathbb{E}\left[\left\|\widehat{\lambda}_{\widehat{m}_{1}}-\lambda\right\|_{F_{T}}^{2} \mathbb{1}_{\Delta_{1} \cap \Delta_{2} \cap \Delta_{3}}\right] \leq C_{1}\left\{\left\|\lambda-\lambda_{m}\right\|_{\bar{F}_{T}}^{2}+\mathbb{E}\left[\left(\text { pen }_{1}(m)-\text { pen }_{1}\left(\widehat{m}_{1}\right)\right.\right.\right. \\
& \left.\left.+2 p_{1}\left(m, \widehat{m}_{1}\right)\right) \mathbb{1}_{\Delta_{3}}\right]+\|\lambda\|_{\bar{F}_{T}}^{2} \frac{1}{\bar{F}_{0} n} \\
& \left.+\sum_{m^{\prime} \in \mathcal{M}_{n}} \mathbb{E}\left[\left(\sup _{t \in S_{m}+S_{m^{\prime}},\|t\|_{\bar{F}_{T}}=1}\left(v_{n, 1}(t)\right)^{2}-\frac{2}{3} p_{1}\left(m, m^{\prime}\right)\right)_{+}\right]\right\}
\end{aligned}
$$

with

$$
p_{1}\left(m, m^{\prime}\right)=\frac{2 B}{5} \frac{K^{2}}{\bar{F}_{0}} \frac{D_{m}+D_{m^{\prime}}}{n} .
$$


The only difference with the proof of Proposition 6.1 is the upper bound of $\mathbb{E}\left[\left(\operatorname{pen}_{1}(m)-\right.\right.$ pen $\left.\left._{1}\left(\widehat{m}_{1}\right)+2 p_{1}\left(m, \widehat{m}_{1}\right)\right) \mathbb{1}_{\Delta_{3}}\right]$. Indeed,

$$
\begin{aligned}
\mathbb{E} & {\left[\left(\text { pen }_{1}(m)-\text { pen }_{1}\left(\widehat{m}_{1}\right)+2 p_{1}\left(m, \widehat{m}_{1}\right)\right) \mathbb{1}_{\Delta_{3}}\right] } \\
= & \mathbb{E}\left[\left(\frac{B}{\widehat{F}_{0}} \frac{D_{m}-D_{\widehat{m}}}{n}+\frac{4 B}{5 \bar{F}_{0}} \frac{D_{m}+D_{\widehat{m}}}{n}\right) \mathbb{1}_{\Delta_{3}}\right] \\
\leq & \mathbb{E}\left[\left(\frac{B}{\widehat{F}_{0}} \frac{D_{m}-D_{\widehat{m}}}{n}+\frac{B}{\widehat{F}_{0}} \frac{D_{m}+D_{\widehat{m}}}{n}\right) \mathbb{1}_{\Delta_{3}}\right] \\
& =\mathbb{E}\left[\frac{2 B}{\widehat{F}_{0}} \frac{D_{m}}{n} \mathbb{1}_{\Delta_{3}}\right] \leq \frac{8 B}{3 \bar{F}_{0}} \frac{D_{m}}{n} .
\end{aligned}
$$

\subsection{Proof of Proposition 7.2}

If $\alpha_{n} \leq \bar{F}_{0} / 2$, then

$$
\begin{aligned}
P\left[\Delta_{3}^{c}\right] & =P\left[\left|\frac{1}{n} \sum_{i=1}^{n}\left(\mathbb{1}_{\left\{T_{i} \geq 1\right\}}-\bar{F}_{0}\right)\right| \geq \frac{1}{4} \bar{F}_{0}\right] \\
& =P\left[\left|\frac{1}{n} \sum_{i=1}^{n}\left(\mathbb{1}_{\left\{T_{i} \geq 1\right\}}-\mathbb{E}\left[\mathbb{1}_{\left\{T_{i} \geq 1\right\}}\right]\right)\right| \geq \frac{1}{4} \bar{F}_{0}\right]
\end{aligned}
$$

We apply Bernstein inequality with the parameters $c=1$ and $v=\bar{F}_{0}$, then $P\left[\Delta_{3}^{c}\right] \leq$ $2 \exp \left(-C_{2} n \bar{F}_{0}\right)$ where $C_{1}$ is a numerical constant.

\subsection{Proof of Proposition 7.3}

Let $x_{0}$ and $\widehat{x}_{0}$ be in $A$ such that

$$
v=\|\lambda\|_{\infty, A}=\lambda\left(x_{0}\right) \text { and } \widehat{v}_{n}=\left\|\widehat{\lambda}_{D}\right\|_{\infty, A}=\widehat{\lambda}_{D}\left(\widehat{x}_{0}\right) .
$$

Then

$$
\begin{aligned}
\widehat{v}_{n}-v \leq\left(\widehat{\lambda}_{D}-\lambda\right)\left(\widehat{x}_{0}\right) & =\left(\widehat{\lambda}_{D}-\lambda_{D}\right)\left(\widehat{x}_{0}\right)+\left(\lambda_{D}-\lambda\right)\left(\widehat{x}_{0}\right) \\
& \leq \sqrt{\frac{D}{a}} \sup _{j=1, \ldots, D}\left|\widehat{a}_{j}-a_{j}\right|+\left\|\lambda-\lambda_{D}\right\|_{\infty}
\end{aligned}
$$

Similarly,

$$
\begin{aligned}
v-\widehat{v}_{n} & \leq\left(\lambda-\widehat{\lambda}_{D}\right)\left(x_{0}\right) \leq\left(\lambda-\lambda_{D}\right)\left(x_{0}\right)+\left(\lambda_{D}-\widehat{\lambda}_{D}\right)\left(x_{0}\right) \\
& \leq\left\|\lambda-\lambda_{D}\right\|_{\infty}+\sqrt{\frac{D}{a}} \sup _{j=1, \ldots, D}\left|\widehat{a}_{j}-a_{j}\right| .
\end{aligned}
$$


Hence $\left|v-\widehat{v}_{n}\right| \leq\|\lambda-\lambda\|_{\infty}+\sqrt{\frac{D}{a}} \sup _{j=1, \ldots, D}\left|\widehat{a}_{j}-a_{j}\right|$, and according to Eq. 14,

$$
\begin{aligned}
P\left[\Delta_{4}^{c}\right] & =P\left[\left|\nu-\widehat{v}_{n}\right|>\frac{1}{4}\right] \\
& \leq P\left[\left\|\lambda-\lambda_{D}\right\|_{\infty}+\sqrt{\frac{D}{a}} \sup _{j=1, \ldots, D}\left|\widehat{a}_{j}-a_{j}\right|>\frac{v}{4}\right] \\
& \leq P\left[\sqrt{\frac{D}{a}} \sup _{j=1, \ldots, D}\left|\widehat{a}_{j}-a_{j}\right|>\frac{v}{8}\right] \leq \sum_{j=1}^{D} P\left[\sqrt{\frac{D}{a}}\left|\widehat{a}_{j}-a_{j}\right|>\frac{v}{8}\right] .
\end{aligned}
$$

Besides, for every $j=1, \ldots, D$,

$$
\begin{aligned}
\sqrt{\frac{D}{a}}\left(\widehat{a}_{j}-a_{j}\right)= & \sqrt{\frac{D}{a}}\left[\frac{(1 / n) \sum_{i=1}^{n} \delta_{i} \varphi_{j}^{D}\left(T_{i}\right)}{\left\|\varphi_{j}^{D}\right\|_{n}^{2}}-\frac{\int_{A} \varphi_{j}^{D}(x) \lambda(x) \bar{F}_{T}(x) d x}{\left\|\varphi_{j}^{D}\right\|_{\bar{F}_{T}(x)}^{2}}\right] \\
= & \left(\frac{1}{\left\|\varphi_{j}^{D}\right\|_{n}^{2}} \sqrt{\frac{D}{a}}\right) \frac{1}{n} \sum_{i=1}^{n}\left[\delta_{i} \varphi_{j}^{D}\left(T_{i}\right)-\int_{A} \varphi_{j}^{D}(x) \lambda(x) \bar{F}_{T}(x) d x\right] \\
& +\sqrt{\frac{D}{a}} \int_{A} \varphi_{j}^{D}(x) \lambda(x) \bar{F}_{T}(x) d x\left[\frac{1}{\left\|\varphi_{j}^{D}\right\|_{n}^{2}}-\frac{1}{\left\|\varphi_{j}^{D}\right\|_{\bar{F}_{T}}^{2}}\right] .
\end{aligned}
$$

Moroever, on the set $\Delta_{1}$,

$$
\left\|\varphi_{j}^{D}\right\|_{n}^{2} \geq \frac{3}{4}\left\|\varphi_{j}^{D}\right\|_{\bar{F}_{T}}^{2}=\frac{3}{4} \int_{a(j-1) / D}^{a j / D} \frac{D}{a} \bar{F}_{T}(x) d x \geq \frac{3 \bar{F}_{0}}{4}
$$

and

$$
\left|\int_{A} \varphi_{j}^{D}(x) \lambda(x) \bar{F}_{T}(x) d x\right| \leq\|\lambda\|_{\bar{F}_{T}}\left\|\varphi_{j}^{D}\right\|_{\bar{F}_{T}} \leq\|\lambda\|_{\bar{F}_{T}} .
$$

Hence

$$
\begin{aligned}
\sqrt{\frac{D}{a}}\left|\widehat{a}_{j}-a_{j}\right| \mathbb{1}_{\Delta_{1}} \leq & \frac{4}{3 \bar{F}_{0}} \sqrt{\frac{D}{a}} \mid \frac{1}{n} \sum_{i=1}^{n}\left[\delta_{i} \varphi_{j}^{D}\left(T_{i}\right)-\int_{A} \varphi_{j}^{D}(x) \lambda(x) \bar{F}_{T}(x) d x \mid\right. \\
& +\sqrt{\frac{D}{a}}\|\lambda\|_{\bar{F}_{T}}\left|\frac{\left\|\varphi_{j}^{D}\right\|_{\bar{F}_{T}}^{2}-\left\|\varphi_{j}^{D}\right\|_{n}^{2}}{\left\|\varphi_{j}^{D}\right\|_{\bar{F}_{T}}^{2}\left\|\varphi_{j}^{D}\right\|_{n}^{2}}\right|
\end{aligned}
$$




$$
\begin{array}{r}
\leq \frac{4}{3 \bar{F}_{0}} \sqrt{\frac{D}{a}}\left|\frac{1}{n} \sum_{i=1}^{n}\left(\delta_{i} \varphi_{j}^{D}\left(T_{i}\right)-\mathbb{E}\left[\delta_{i} \varphi_{j}^{D}\left(T_{i}\right)\right]\right)\right| \\
+\sqrt{\frac{D}{a}}\|\lambda\|_{\bar{F}_{T}} \frac{4^{2}}{3^{2} \bar{F}_{0}^{2}}\left|\left\|\varphi_{j}^{D}\right\|_{\bar{F}_{T}}^{2}-\left\|\varphi_{j}^{D}\right\|_{n}^{2}\right| .
\end{array}
$$

Thus

$$
\begin{aligned}
P\left[\Delta_{4}^{c} \cap \Delta_{1}\right] \leq & \sum_{j=1}^{D} P\left[\frac{4}{3 \bar{F}_{0}} \sqrt{\frac{D}{a}}\left|\frac{1}{n} \sum_{i=1}^{n}\left(\delta_{i} \varphi_{j}^{D}\left(T_{i}\right)-\mathbb{E}\left[\delta_{i} \varphi_{j}^{D}\left(T_{i}\right)\right]\right)\right| \geq \frac{v}{16}\right] \\
& +\sum_{j=1}^{D} P\left[\sqrt{\frac{D}{a}}\|\lambda\|_{\bar{F}_{T}} \frac{4^{2}}{3^{2} \bar{F}_{0}^{2}}\left|\left\|\varphi_{j}^{D}\right\|_{\bar{F}_{T}}-\left\|\varphi_{j}^{D}\right\|_{n}^{2}\right| \geq \frac{v}{16}\right] \\
= & \sum_{j=1}^{D}\left(P_{1, j}+P_{2, j}\right) .
\end{aligned}
$$

$P_{1, j}$ and $P_{2, j}$ are upper bounded with Bernstein Inequality. For $P_{1, j}$, the parameters $b$ and $v$ are the following.

$$
\begin{gathered}
\mathbb{E}\left[\delta_{i}^{2}\left(\varphi_{j}^{D}\right)^{2}\left(T_{i}\right)\right]=\int_{A}\left(\varphi_{j}^{D}\right)^{2}(x) \lambda(x) \bar{F}_{T}(x) d x \leq\|\lambda\|_{\infty, A}=v \\
\text { and }\left\|\delta_{i} \varphi_{j}^{D}\left(T_{i}\right)\right\|_{\infty} \leq \sqrt{\frac{D}{a}}=c .
\end{gathered}
$$

Hence, for every $j \in\{1, \ldots, D\}$,

$$
P_{1, j} \leq 2 \exp \left(-C \frac{n}{D}\right)
$$

where $C$ depends on $\left(\nu,\|\lambda\|_{\infty, A}, \bar{F}_{0}, a\right)$. Let us upper bound $P_{2, j}$. For every $j \in$ $\{1, \ldots, D\}$,

$$
\begin{aligned}
P_{2, j}=P & {\left[\frac{4^{2}\|\lambda\|_{\bar{F}_{T}}}{3^{2} \bar{F}_{0}^{2}} \sqrt{\frac{D}{a}} \mid \frac{1}{n} \sum_{i=1}^{n}\left(\int_{A}\left(\varphi_{j}^{D}\right)^{2}(x) \mathbb{1}_{\left\{T_{i} \geq x\right\}} d x\right.\right.} \\
& \left.\left.-\mathbb{E}\left[\int_{A}\left(\varphi_{j}^{D}\right)^{2}(x) \mathbb{1}_{\left\{T_{i} \geq x\right\}} d x\right]\right) \mid \geq \frac{v}{16}\right]
\end{aligned}
$$


and

$\mathbb{E}\left[\left(\int_{A}\left(\varphi_{j}^{D}\right)^{2}(x) \mathbb{1}_{\left\{T_{i} \geq x\right\}} d x\right)^{2}\right] \leq 1=v$ and $\left\|\int_{A}\left(\varphi_{j}^{D}\right)^{2}(x) \mathbb{1}_{\left\{T_{i} \geq x\right\}} d x\right\|_{\infty} \leq 1=c$.

Thus, with Bernstein inequality we obtain

$$
P_{2, j} \leq 2 \exp \left(-C^{\prime} \frac{n}{D}\right)
$$

where $C^{\prime}$ depends on $\left(v,\|h\|_{\bar{F}_{T}}, \bar{F}_{0}, a\right)$. Then Eqs. 26, 27 and 28 conclude the proof of Proposition 7.3.

\section{Appendix}

Lemma 8.1 Let $M$ be a symmetric matrix of dimension $n$, with real coefficients, then

$$
\min (S p(M))=\min _{\left\{U \in \mathbb{R}^{n}, U \neq 0\right\}} \frac{U^{t} M U}{U^{t} U} .
$$

Proof of Lemma 8.1 $M$ is a real symmetric matrix hence according to classical algebra results, there exist an orthogonal matrix $P$ and a diagonal matrix $D$ such that $M=P^{t} D P$. Moreover, $D=\operatorname{diag}\left(\lambda_{1}, \ldots, \lambda_{n}\right)$ where $\lambda_{j}$ is an eigenvalue of $M$ for every $j \in\{1, \ldots, n\}$. Let $j_{0}=\arg \min _{j=1, \ldots n} \lambda_{j}$.

On the one hand, let $U \in \mathbb{R}^{n}$, and $V=P U=\left(v_{1}, \ldots, v_{n}\right)^{t}$, then

$$
U^{t} M U=(P U)^{t} D(P U)=\sum_{j=1}^{n} \lambda_{j} v_{j}^{2} \geq \min (S p(M)) \sum_{j=1}^{n} v_{j}^{2}=\min (S p(M)) V^{t} V
$$

Thus $\min (\operatorname{Sp}(M)) \leq \min _{\left\{U \in \mathbb{R}^{n}, U \neq 0\right\}} \frac{U^{t} M U}{U^{t} U}$.

On the other hand, Let $V_{0}$ be the vector whose coordinates are all zero except the $j_{0}^{\text {th }}$ which is equal to 1 . Let $U_{0}=P^{-1} V_{0}$, then $U_{0}^{t} U_{0}=V_{0}^{t} V_{0}=1$ since $P$ is orthogonal.

$$
U_{0}^{t} M U_{0}=V_{0}^{t} D V_{0}=\lambda_{j_{0}}=\min (\operatorname{Sp}(M)) U_{0}^{t} U_{0}
$$

hence $\min (S p(M))=\min _{\left\{U \in \mathbb{R}^{n}, U \neq 0\right\}} \frac{U^{t} M U}{U^{t} U}$.

The following inequality, called Bernstein Inequality, is proved in Birgé and Massart (1998), p.366, Lemma 8.

Theorem 3 Let $\left(V_{1}, \ldots, V_{n}\right)$ be independent random variables. Assume that

$$
\frac{1}{n} \sum_{i=1}^{n} \mathbb{E}\left[V_{i}^{2}\right] \leq v \quad \text { and } \quad \frac{1}{n} \sum_{i=1}^{n} \mathbb{E}\left[\left(V_{i}\right)_{+}^{l}\right] \leq \frac{l !}{2} \times v \times c^{l-2}
$$


for every $l \geq 2$. Let $S=(1 / n) \sum_{i=1}^{n} X_{i}-\mathbb{E}\left[V_{i}\right]$.

(1) For every $\epsilon>0$,

$$
P[S \geq \sqrt{2 v x}+c x] \leq \exp (-n x) \text { and } P[|S| \geq \sqrt{2 v x}+c x] \leq 2 \exp (-n x) .
$$

(2) Similarly, for every $\epsilon>0$,

$$
P[S \geq \epsilon] \leq \exp \left(-\frac{n \epsilon^{2}}{2(v+c \epsilon)}\right) \quad \text { and } \quad P[|S| \geq \epsilon] \leq 2 \exp \left(-\frac{n \epsilon^{2}}{2(v+c \epsilon)}\right)
$$

Theorem 4 Let $\mathcal{F}$ be a set of uniformly bounded functions, which have a countable dense sub-family for the infinite norm subspace. Let $\left(V_{1}, \ldots, V_{n}\right)$ be independent random variables and

$$
Z=\sup _{f \in \mathcal{F}}\left|\frac{1}{n} \sum_{i=1}^{n}\left[f\left(V_{i}\right)-\mathbb{E}\left(f\left(V_{i}\right)\right)\right]\right|
$$

Consider $b$, $v$ and $\mathbb{H}$ such that

$$
b \geq \sup _{f \in \mathcal{F}}\|f\|_{\infty}, \quad v \geq \sup _{f \in \mathcal{F}} \frac{1}{n} \sum_{i=1}^{n} \operatorname{Var}(f(V i)) \quad \text { and } \quad \mathbb{H} \geq \mathbb{E}(Z) .
$$

Then for every $\theta>1$, there exist numerical constants $\bar{C}, \bar{C}^{\prime}, \bar{\kappa}, \bar{\kappa}^{\prime}$ such that

$$
\mathbb{E}\left[\left(Z^{2}-\theta \mathbb{H}^{2}\right)_{+}\right] \leq \bar{C} \frac{v}{n} \exp \left(-\bar{\kappa} \frac{n \mathbb{H}^{2}}{v}\right)+\bar{C}^{\prime} \frac{b^{2}}{n^{2}} \exp \left(-\bar{\kappa}^{\prime} \frac{n \mathbb{H}}{b}\right)
$$

The above version of Talagrand Inequality is enunciated for example in Lacour (2008) (Sect. 6, Lemma 5).

\section{References}

Andersen PK, Borgan O, Gill R, Kieding N (1993) Statistical models based on counting processes. Springer, Berlin

Antoniadis A, Grégoire G, Nason G (1999) Density hazard rate estimation for right-censored data by using wavelet methods. J R Statist Soc B 61(1):63-84

Bagkavos D, Patil P (2009) Variable bandwidths for nonparametric hazard rate estimation. Comm Statist Theory Methods 38(6-7):1055-1078

Baraud Y (2002) Model selection for regression on a random design. ESAIM Probab Statist 6:127-146

Birgé L, Massart P (1998) Minimum contrast estimators on sieves: exponential bounds and rates of convergence. Bernoulli 4(3):329-375

Brunel E, Comte F (2005) Penalized contrast estimation of density and hazard rate with censored data. Sankhya 67(3):441-475

Brunel E, Comte F (2008) Adaptive estimation of hazard rate with censored data. Comm Statist Theory Methods 37(8-10):1284-1305

Efromovich S (2007) Optimal nonparametric estimation of the density of regression errors with finite support. Ann Inst StatistMath 59(4):617-654 
Huber C, MacGibbon B (2004) Lower bounds for estimating a hazard, Handbook of statistics, vol 23. Elsevier, Amsterdam, pp 209-226

Kaplan EL, Meier P (1958) Non parametric estimation from incomplete observations. J Amer Statist Assoc 53:457-481

Lacour C (2008) Adaptive estimation of the transition density of a particular hidden markov chain. J Multivariate Anal 99(5):787-814

Muller HG, Wang J (1994) Hazard rate estimation under random censoring with varying kernels and bandwidth. Biometrics 50(1):61-76

Nelson W (1972) Theory and applications of hazard plotting for censored failure data. Technometrics 14(4):945-966

Nielsen JP (2003) Variable bandwidth kernel hazard estimators. Nonparam Stat 15(3):355-376

Patil PN (1993) On the least squares cross validation bandwidth in hazard rate estimation. Ann Statist 21(4):1792-1810

Reynaud-Bouret P (2006) Penalized projection estimators of the Aalen multiple intensity. Bernoulli 12(4):633-661

Tanner MA, Wong WH (1983) The estimation of the hazard function from randomly censored data by the kernel method. Ann Statist 11(3):989-993. doi:10.1214/aos/1176346265

Yandell BS (1983) Nonparametric inference for rates with censored survival data. Ann Satist 11(4):11191135 